\title{
EMISIÓN DIFUSA DE CO, Y ACTIVIDAD VOLCÁNICA EN EL VOLCÁN POÁS, COSTA RICA
}

\author{
DIFFUSE CO, EMISSION AND VOLCANIC ACTIVITY AT POÁS VOLCANO, \\ COSTA RICA, CENTRAL AMERICA
}

\section{Gladys V. Melián ${ }^{1 *}$, Nemesio M. Pérez ${ }^{1}$, Pedro A. Hernández ${ }^{1}$, Dacil Nolasco ${ }^{1}$, Rayco Marrero ${ }^{1}$, Mario Fernández ${ }^{2}$, Carlos Ramírez ${ }^{2}$, Raúl Mora-Amador ${ }^{3}$ \& Guillermo E. Alvarado ${ }^{2,3}$}

${ }^{1}$ División de Medio Ambiente, Instituto Tecnológico y de Energías Renovables (ITER). Apdo postal: 38611 Granadilla, S/C de Tenerife, España ${ }^{2}$ Centro de Investigación en Ciencias Geológicas (CICG), Universidad de Costa Rica (UCR). Apdo postal: 1000 San José, Costa Rica ${ }^{3}$ Escuela Centroamericana de Geología, Universidad de Costa Rica (UCR). Apdo postal: 1000 San José, Costa Rica

*Autora para contacto: gladys@iter.es

(Recibido: 15/08/2009; aceptado: 08/11/2010)

\begin{abstract}
Four concentration and soil efflux $\mathrm{CO}_{2}$ surveys were undertaken in the period 2000-2003 at Poás volcano, with the aim of evaluating the total diffuse $\mathrm{CO}_{2}$ emission and investigating their spatial distributions. The $\mathrm{CO}_{2}$ concentration and total diffuse emission showed a minimum value at 2000, followed by a significant increase at 2001 and finally a decrease at 2003 study. The spatial distribution of soil $\mathrm{CO}_{2}$ efflux and the $\delta^{13} \mathrm{C}$ isotopic composition in the $\mathrm{CO}_{2}$ showed a good agreement with the location of the main volcanic-structural characteristics of the area, with the main $\mathrm{CO}_{2}$ emission anomalies observed close to the fumarolic fields and hot springs. A magmatic intrusion during occurred in the period 1998-2004 at Poás might be the responsible of the observed $\mathrm{CO}_{2}$ emission changes. Monitoring diffuse $\mathrm{CO}_{2}$ emission has became an important geochemical tool for the monitoring of Poás volcanic activity.

Keywords: $\mathrm{CO}_{2}$ concentration, diffuse $\mathrm{CO}_{2}$ emissions, $\delta^{13} \mathrm{C}$ isotopic composition, Poás.

RESUMEN: Entre 2000 y 2003, se realizaron cuatro estudios de la concentración y flujo difuso de $\mathrm{CO}_{2}$ en el Poás, con el fin de investigar la distribución espacial y evaluar la emisión total de $\mathrm{CO}_{2}$ a la atmósfera por este sistema. La evolución temporal de la concentración y flujo difuso de $\mathrm{CO}_{2}$ muestra un mínimo en la emisión en el año 2000 seguida de un
\end{abstract}


incremento de la concentración y el flujo difuso de $\mathrm{CO}_{2}$ en el 2001 y 2002, y un decrecimiento en 2003. La distribución espacial de las anomalías de flujo difuso de $\mathrm{CO}_{2}$, y de la composición química e isotópica del gas del suelo muestra una correlación positiva con las principales características volcánico-estructurales del Poás. Los cambios observados parecen estar relacionados con una intrusión magmática ocurrida en el Poás entre 1998-2004. Este estudio muestra la importancia de la geoquímica importante para la vigilancia volcánica del Poás.

Palabras clave: Concentración de $\mathrm{CO}_{2}$, emisión difusa de $\mathrm{CO}_{2}$, Composición isotópica $\delta^{13} \mathrm{C}$, Poás, Costa Rica.

\section{INTRODUCCIÓN}

Los volcanes son las principales ventanas de desgasificación del planeta. La emisión de gases por los sistemas volcánicos ocurre tanto durante períodos eruptivos como en períodos de calma volcánica. El hecho de que los gases disueltos en el magma son los que proporcionan la fuerza motriz de las erupciones volcánicas, ha permitido que en la actualidad el estudio y seguimiento de la composición química e isotópica de los gases volcánicos así como de sus emisiones a la atmósfera sean un instrumento muy útil para la monitorización y vigilancia de la actividad volcánica (Matsuo, 1960; Matsuo et al., 1975; Casadevall et al., 1983; Hirabayashi et al., 1986; Sato, 1988; Symonds et al., 1990; Sano et al., 1991; Casadevall et al., 1994; Giggenbach, 1996; Chiodini et al., 1998; McGee \& Gerlach, 1998; Hernández et al., 2001a; Carapezza et al., 2004; Pérez et al., 2006; Pérez \& Hernández, 2007; Gurrieri et al., 2008; Rizzo et al., 2009). Durante las dos últimas décadas, los estudios de emisión difusa de $\mathrm{CO}_{2}$ por los volcanes han sido numerosos, poniendo de manifiesto la importancia de esta metodología para evaluar la dinámica de desgasificación de los volcanes y su relación con la actividad volcánica (Carbonelle et al., 1985; Allard et al., 1987, 1991; Baubron et al., 1990, 1991; Pérez, 1992; Farrar et al., 1995; Giammanco et al., 1995; Chiodini et al., 1996; Pérez et al., 1996, 2004, 2006; Hernández et al., 1998, 2001b; Salazar et al., 2001, 2003; Melián et al., 2004; Granieri et al., 2006; Lecinsky et al., 2007; Padrón et al., 2008; Rizzo et al., 2009).

La mayoría de los estudios realizados en el ámbito de desgasificación difusa hacen referencia al $\mathrm{CO}_{2}$ por ser la segunda especie mayoritaria de los gases volcánicos después del vapor de agua y por su baja solubilidad en fundidos silicatados (Gerlach \& Graeber, 1985) así como por la facilidad para ser medido in situ (Allard et al., 1987, 1991; Baubron et al., 1991; Pérez, 1992; Farrar et al., 1995; Giammanco et al., 1995; Pérez et al., 1996, 2004, 2006; Hernández et al., 1998, 2001a, 2001b, 2003, 2006; Salazar et al., 2001, 2003; Cardellini et al, 2003; Frondini et al., 2004; Melián et al., 2004; Notsu et al., 2005; Padrón et al., 2008).

En este trabajo se presenta un estudio de la composición química e isotópica $\left(\delta^{13} \mathrm{C}\right) \operatorname{del} \mathrm{CO}_{2} \mathrm{y}$ del flujo difuso de $\mathrm{CO}_{2}$ en el ambiente superficial del Poás con el fin de evaluar la distribución espacial de las emisiones de $\mathrm{CO}_{2}$ y su variabilidad en el tiempo, así como cuantificar la emisión de $\mathrm{CO}_{2}$ a la atmósfera por este volcán durante el periodo de estudio 2000-2003 y su relación con la actividad volcánica. En este estudio se pone de manifiesto la importancia de la vigilancia volcánica mediante el seguimiento geoquímico de los gases volcánicos para evaluar el estado de actividad del Poás.

\section{GEOLOGÍA DEL ÁREA DE ESTUDIO}

El Poás es un estratovolcán andesítico-basáltico de $2708 \mathrm{~m}$ de altitud perteneciente a la cadena volcánica de América Central. La actividad volcánica en América Central está íntimamente relacionada con la actividad tectónica, debida a la subducción de la placa de Cocos en la placa Caribe. El Poás es un estratovolcán complejo $\left(300 \mathrm{~km}^{2}\right)$, altamente vegetado y compuesto por tres cráteres alineados en dirección N-S; el cono Von Franzius, el cráter principal y el cono Botos (Fig. 1). El cráter principal es de forma subcónica irregular con 

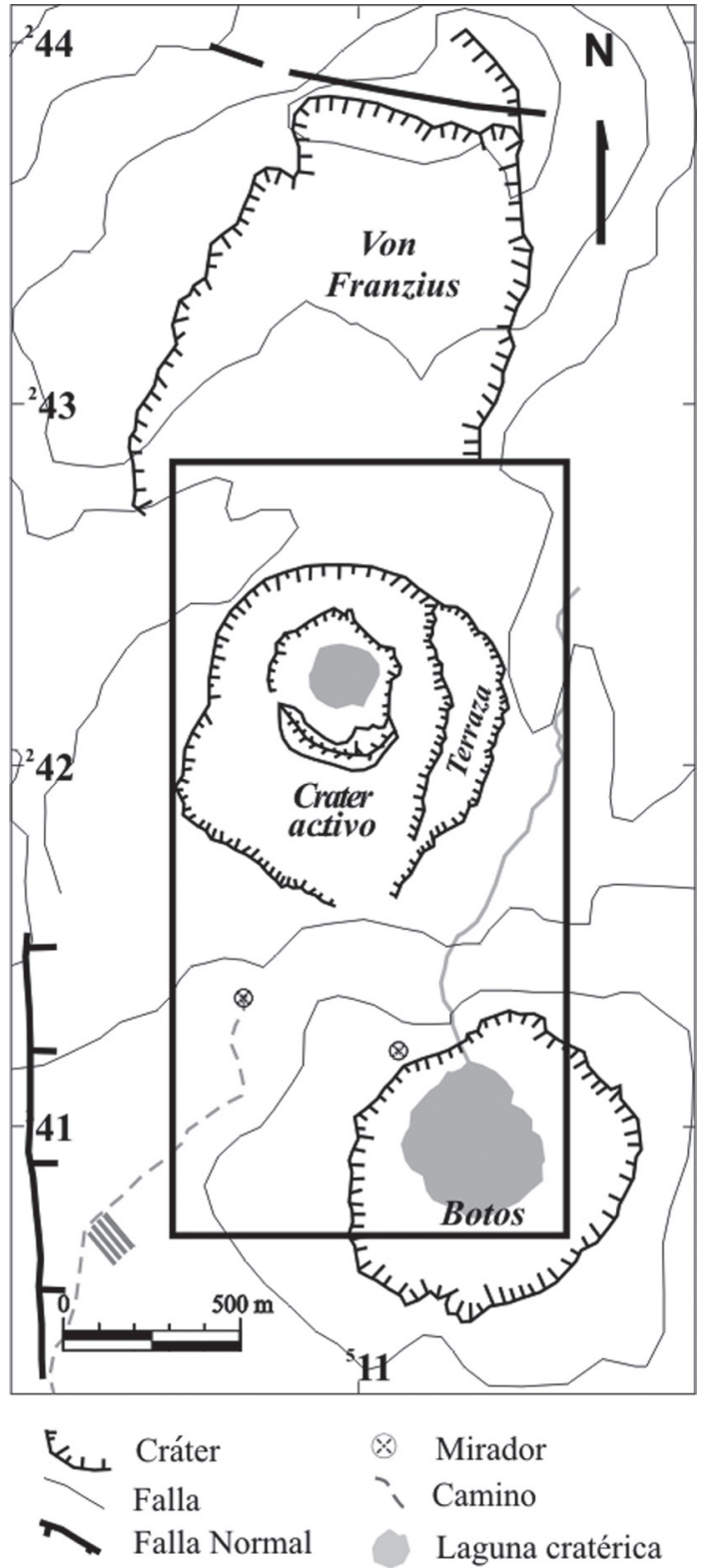

Fig. 1: Mapa del cráter activo del volcán Poás y alrededores donde se muestra la zona de estudio.

$1,5 \mathrm{~km}$ diámetro y $320 \mathrm{~m}$ de profundidad, y es donde históricamente se ha centrado la actividad volcánica. Entre 1953-1955, tiene lugar la formación de las dos principales particularidades del cráter principal del Poás (Casertano et al., 1985, 1987; Rowe et al., 1992a). Durante esta erupción existen dos ventanas abiertas en el interior del cráter principal, en una de ellas se desarrolla el cono de escorias que es denominado generalmente domo, localizado en la parte central del cráter y la otra a unos $150 \mathrm{~m}$ al $\mathrm{N}$ del domo que colapsa y forma de nuevo la Laguna Caliente. La Laguna Caliente del Poás es altamente ácida $(\mathrm{pH} \sim 0)$ tiene un diámetro de $300 \mathrm{~m}$ y una temperatura entre 40 y $60^{\circ} \mathrm{C}$ cuyo volumen, temperatura y propiedades químicas varían con el régimen pluviométrico y el grado de actividad volcánica (Brown et al., 1989; Rowe et al., 1992a, 1992b; Rymer \& Brown, 1989; Martínez et al., 2000). La Laguna Caliente del Poás actúa como un medio amortiguador entre el sistema hidrotermal del volcán y la atmósfera, por lo que según Pasternack \& Varekamp (1997), la laguna puede ser descrita como "altamente activa". El domo se eleva a unos $40 \mathrm{~m}$ sobre la laguna presentando en el flanco norte numerosas fumarolas activas de temperatura e intensidad variable. Los gases que emiten estas fumarolas, unido a los aerosoles emitidos por la laguna son los que dan origen a la característica pluma del volcán.

Al norte del cráter principal se encuentra el cono Von Franzius que se eleva a unos $2639 \mathrm{~m}$ s.n.m. Constituye un centro eruptivo viejo del Poás que en la actualidad se encuentra inactivo y algo erosionado. El otro cono presente en la estructura es el denominado Botos, de $2708 \mathrm{~m}$ s.n.m. Está localizado aproximadamente a $1 \mathrm{~km}$ al suroeste del cráter más activo, presentando un cráter de unos $750 \mathrm{~m}$ de diámetro. Dentro de este, se encuentra una laguna fría de aproximadamente $400 \mathrm{~m}$ de diámetro y de $15 \mathrm{~m}$ de profundidad en su centro y con temperaturas entre 11 y $18^{\circ} \mathrm{C}$ (Rymer et al., 2000) cuya agua es de origen meteórico.

La actividad sísmica es una de las principales características del Poás ya que se ha observado una estrecha relación entre la sismicidad y la actividad volcánica (Fernández, 1990). El Poás se encuentra vigilado sísmicamente desde 1974 por la Red Sismológica Nacional (RSN. ICE-UCR) con una estación simple de periodo corto localizada cerca del cráter principal (Fernández, 1990). La sismicidad del Poás se caracteriza por presentar sismos tipo A (señales de alta frecuencia $>3,0 \mathrm{~Hz}$ ), sismos tipo B (señales de baja frecuencia $<3,0 \mathrm{~Hz}$ ) y tremor volcánico (Minakami, 1969). Durante 2000 y 2003, periodo en que se realiza el estudio 
de desgasificación difusa en el Poás, se han observado variaciones de la actividad sísmica volcanotectónica o Tipo A, relacionada con movimientos de magma a través de fracturas y/o cambios de estrés en el sistema volcánico-hidrotermal del Poás. Estos movimientos implican cambios en las condiciones físico-químicas y por tanto un aporte adicional de gases a la superficie, ya que cambian las condiciones del sistema hidrotermal. Durante el periodo de estudio la sismicidad tipo A ha sido variable, con picos notables entre septiembrenoviembre de 1999, marzo-agosto y octubre-diciembre de 2001 y febrero-mayo de 2003 (Melián et al, 2007). La sismicidad Tipo B fue bastante alta durante todo el periodo de estudio de desgasificación difusa y se mantuvo relativamente constante con un pico significativo en 2001 y son normalmente considerados indicadores de la actividad hidrotermal del sistema volcánico del Poás. Las variaciones de la actividad sísmica del Poás actúan como generadores de cambios en la emisión de gases y volátiles procedentes del sistema volcánico-hidrotermal (Martínez et al., 2000) por lo que son importante para explicar los cambios ocurridos en la desgasificación difusa del sistema durante el periodo de estudio.

Los estudios de microgravimetría realizados en el volcán Poás durante los últimos años (Rymer et al., 2000, 2005; Locke et al., 2003; Fournier et al., 2004) relacionan cambios significativos de gravimetría con movimientos de magma en el sistema volcánico-hidrotermal del Poás. Entre 2000 y 2003 , periodo en que se realizó el estudio de desgasificación difusa en el Poás, se observaron cambios gravimétricos significativos. Estas variaciones en los valores de microgravimetría en el Poás entre 1998 y 2004 son interpretadas por Rymer et al. (2005) como una intrusión detrítica localizada al oeste del cráter principal que genera celdas convectivas en el interior que pueden cambiar o generarse en un intervalo de 5 años y drenar cada 7-10 años.

\section{METODOLOGÍA}

Entre los meses de febrero-mayo de 2000, 2001, 2002 y 2003, se realizaron cuatro campañas de flujo difuso y concentración de $\mathrm{CO}_{2}$ en el cráter activo del volcán Poás y alrededores. La selección de los puntos de muestreo se realizó teniendo en cuenta las características geológicas y estructurales del área de estudio, así como de accesibilidad, con el fin de obtener una buena distribución espacial. El área de estudio, $3,2 \mathrm{~km}^{2}$, fue la misma para los cuatro estudios. Los estudios de desgasificación difusa de $\mathrm{CO}_{2}$ realizados en el periodo 2000-2003 coincidieron siempre con la estación seca. El número de puntos de medida y toma de muestras fue de 109, 182, 244 y 238 para $2000,2001,2002$ y 2003, respectivamente. Las medidas de flujo difuso de $\mathrm{CO}_{2}$ se realizaron siguiendo el método de la cámara de acumulación (Parkinson, 1981; Chiodini et al., 1996). Este método consiste en depositar sobre la superficie del suelo una cámara cilíndrica abierta por un extremo que permite la acumulación del gas proveniente del suelo. El gas acumulado es bombeado a través de un circuito cerrado hacia el detector NDIR (Infrarrojo No-Dispersivo) de $\mathrm{CO}_{2}$, tras el cual el gas regresa a la cámara de acumulación. La salida analógica es transformada convenientemente por un transformador analógico-digital que se conecta a un ordenador portátil el cual permite visualizar la curva de concentración versus tiempo. Nosotros estimamos un error en un $10 \%$ para las medidas del flujo difuso de $\mathrm{CO}_{2}$ en un rango de 10-35000 $\mathrm{g} \mathrm{m}^{-2} \mathrm{~d}^{-1}$ basado en la variabilidad de las réplicas realizadas en el laboratorio con un flujo de $\mathrm{CO}_{2}$ conocido.

En cada punto de observación de las cuatro campañas realizadas se determinó la composición química e isotópica del gas del suelo. Para ello se recogió una alícuota de gas del suelo mediante una sonda de acero inoxidable de $3 \mathrm{~mm}$ de diámetro interno que se inserta a $40-50 \mathrm{~cm}$ de profundidad. La toma de muestra del gas se realizó extrayendo $30 \mathrm{~cm}^{3}$ de gas del suelo con una jeringuilla hipodérmica y transfiriendo una alícuota de este posteriormente a un vacutainer de vidrio sellado de $10 \mathrm{~cm}^{3}$. Para la determinación química del gas del suelo, se utilizó una variante del método descrito por Hinkle \& Kilburn (1979), que consiste en introducir la muestra de gas en un vacutainer de vidrio sellado y llenado previamente con agua, de tal forma que el gas desplaza al agua del vacutainer. Con este método evitamos que los gases más 
ligeros se escapen a través de las paredes del tapón del tubo debido a la cámara de agua que queda en el interior después de introducir la muestra. Para la toma de muestra de la composición isotópica del gas del suelo, el gas se introduce en un vial al que previamente se le ha realizado el vacío.

El análisis de la composición química del gas muestreado se realizó diariamente en el laboratorio de campo con un Micro-cromatógrafo de gases VARIAN modelo 2002P de doble columna. Para la determinación del contenido de $\mathrm{CO}_{2}$ en el gas del suelo se empleó una columna Poraplot-Q de $10 \mathrm{~m}$ de longitud, un detector de conductividad térmica (TDC) y helio $(99,999 \%)$ como gas portador. El límite de detección para el $\mathrm{CO}_{2}$ fue de $68 \mathrm{ppm}$ y la exactitud 2,5\%. En esta columna también se determinó la concentración de metano $\left(\mathrm{CH}_{4}\right)$ en el gas del suelo. En la otra columna (MolSieve $5 \AA$ Á, de $20 \mathrm{~m}$ longitud, TDC y argón como gas portador) se determinó la concentración de $\mathrm{He}, \mathrm{H}_{2}, \mathrm{O}_{2}$ y $\mathrm{N}_{2}$ en el gas del suelo. La composición isotópica del carbono en el gas del suelo fue determinada con un espectrómetro de masas Finnigan MAT MAT Delta-S en el Laboratorio de Química de Terremotos de la Universidad de Tokio, Japón. La relación ${ }^{13} \mathrm{C} /{ }^{12} \mathrm{C}$ se expresa como $\delta^{13} \mathrm{C}$ en partes por mil (\%o) con respecto a un estándar internacional de carbonato V-PBD. El error analítico asociado con la medida de las relaciones isotópicas ${ }^{13} \mathrm{C} /{ }^{12} \mathrm{C}$ fue de $\pm 0,1 \%$.

\section{RESULTADOS Y DISCUSIÓN}

En la Tabla 1 se presenta un resumen estadístico descriptivo de los resultados obtenidos. Los datos fueron tratados usando métodos estadísticos y geoestadísticos. El rango de flujo difuso de $\mathrm{CO}_{2}$ medido en los cuatro estudios se encuentra entre valores por debajo del límite de detección del instrumento $\left(<0,5 \mathrm{~g} \mathrm{~m}^{-2} \mathrm{~d}^{-1}\right)$ y $4712 \mathrm{~g}$ $\mathrm{m}^{-2} \mathrm{~d}^{-1}$ (valor medio de 56, 88, 185 y $76 \mathrm{~g} \mathrm{~m}^{-2} \mathrm{~d}^{-1}$ para 2000, 2001, 2002 y 2003, respectivamente), mientras que los valores de concentración de $\mathrm{CO}_{2}$ en los cuatro estudios se encuentra entre los $360 \mathrm{ppm}$ (concentración de $\mathrm{CO}_{2}$ en el aire) y $80,9 \% \mathrm{~V}$ (valor medio de 0,$92 ; 1,9 ; 2,2$ y 0,22 $\%$ V para 2000, 2001, 2002 y 2003, respectiva- mente). Con el objetivo de investigar si los datos provienen de una distribución unimodal o polimodal y de esta forma identificar la existencia de diferentes poblaciones geoquímicas que nos permite conocer el origen del la concentración y el flujo difuso de $\mathrm{CO}_{2}$, se realizó un análisis estadístico-gráfico en el total de los datos obtenidos (Tennant \& White, 1959; Sinclair, 1974). Los datos de concentración y flujo de $\mathrm{CO}_{2}$ en cada uno de los estudios fueron representados siguiendo una distribución log-normal, mostrando dos poblaciones geoquímicas normales (normal I y normal II): fondo y pico. Los parámetros que caracterizan cada población son calculados mediante el estimador t-Sichel (David, 1977) y se presentan en las Tablas 2 y 3 para la concentración y flujo difuso de $\mathrm{CO}_{2}$, respectivamente. Debido a la alta variabilidad de la población de fondo para la concentración de $\mathrm{CO}_{2}$ y de la población pico de flujo de $\mathrm{CO}_{2}$ en el estudio de 2002 , no se ha podido calcular intervalo de confianza basado en el estimador t-Sichel (David, 1977). Los valores de la población de fondo para el flujo difuso de $\mathrm{CO}_{2}$ variaron entre 1,3 y 14,8 $\mathrm{g} \mathrm{m}^{-2} \mathrm{~d}^{-1}$ durante los cuatro estudios realizados, siendo similares a los valores estimados para la actividad biogénica en otros sistemas volcánicos (Chiodini et al., 1996, 2001; Chiodini y Frondini, 2001; Hernández et al., 2001a, 2001b, 2003, 2006; Frondini et al., 2004; Notsu et al., 2005; Melián, 2008; Padrón et al., 2008). Los valores medios de las poblaciones pico fueron dos ordenes de magnitud superior a los valores de fondo (entre 354 y $4498 \mathrm{~g} \mathrm{~m}^{-2} \mathrm{~d}^{-1}$, media de $\left.2128 \mathrm{~g} \mathrm{~m}^{-2} \mathrm{~d}^{-1}\right)$. Los valores de fondo de la concentración de $\mathrm{CO}_{2}$ fueron estimados entre 605 y $388 \mathrm{ppm}$ (valor medio $495 \mathrm{ppm}$ ), valores similares a la concentración de $\mathrm{CO}_{2}$ en el aire, mientras que los valores de la población pico estimados en los cuatro estudios variaron entre $0,80 \mathrm{y}$ $42 \% \mathrm{~V}$. La existencia de más de una población geoquímica para la concentración y el flujo de $\mathrm{CO}_{2}$ sugieren la presencia de una contribución de origen profundo en el proceso de desgasificación del ambiente superficial del Poás.

Con el fin de comparar los valores de concentración y flujo de $\mathrm{CO}_{2}$ durante el periodo de estudio y estimar la emisión total de $\mathrm{CO}_{2}$ a la 
Cuadro 1

Estadística descriptiva simple de los parámetros físico-químicos estudiados en las campañas de 2000, 2001, 2002 y 2003 en el volcán Poás, Costa Rica

\begin{tabular}{|c|c|c|c|c|c|c|c|c|c|c|c|c|}
\hline & & 2000 & & & 2001 & & & 2002 & & & 2003 & \\
\hline & Min. & Max. & Med. & Min. & Max. & Med. & Min. & Max. & Med. & Min. & Max. & Med. \\
\hline $\begin{array}{c}\mathrm{He} \\
(\mathrm{ppmV})\end{array}$ & 4,2 & 6,2 & 5,6 & 4,4 & 6,0 & 5,4 & 4,8 & 6,1 & 5,8 & 3,5 & 7,6 & 6,3 \\
\hline $\begin{array}{c}\mathrm{H}_{2} \\
(\mathrm{ppmV})\end{array}$ & 0,5 & 1,6 & 0,7 & 0,4 & 1910 & 20,5 & 1,0 & 7059 & 94,9 & 0,2 & 271 & 7,4 \\
\hline $\mathrm{O}_{2}(\% \mathrm{~V})$ & 20,7 & 21,5 & 19,5 & 12,3 & 23,2 & 21,0 & 0,3 & 21,5 & 20,1 & 18,7 & 22,9 & 19,8 \\
\hline $\mathrm{N}_{2}(\% \mathrm{~V})$ & 78,1 & 80,6 & 79,6 & 41,7 & 80,5 & 78,0 & 74,0 & 81,7 & 77,6 & 70,7 & 82,8 & 79,3 \\
\hline $\mathrm{CO}_{2}(\% \mathrm{~V})$ & 0,03 & 58,5 & 0,92 & 0,03 & 53,9 & 1,9 & 0,03 & 80,9 & 2,2 & 0,03 & 4,28 & 0,22 \\
\hline $\begin{array}{l}\text { Flujo } \mathrm{CO}_{2} \\
\left(\mathrm{~g} \mathrm{~m}^{-2} \mathrm{~d}^{-1}\right)\end{array}$ & $<0,5$ & 2361 & 56 & $<0,5$ & 2129 & 88 & $<0,5$ & 2145 & 185 & $<0,5$ & 4712 & 76 \\
\hline$\delta^{13} \mathrm{C}(\% 0)$ & $-52,8$ & $-3,97$ & $-30,1$ & $-65,6$ & 0,92 & $-27,0$ & $-50,9$ & 0,74 & $-22,9$ & n.m. & n.m. & n.m. \\
\hline $\begin{array}{c}\text { T suelo } \\
\left({ }^{\circ} \mathrm{C}\right)\end{array}$ & 7,2 & 92,7 & 17,9 & 8,3 & 92,0 & 18,1 & 10,0 & 94,3 & 18,1 & 10,4 & 93,3 & 19,4 \\
\hline
\end{tabular}

n.m.: no medido

\section{Cuadro 2}

Resumen del análisis estadístico-gráfico aplicado a los datos de concentración de $\mathrm{CO}_{2}$ en el gas del suelo del volcán Poás

\begin{tabular}{|c|c|c|c|c|c|}
\hline Campaña & $\begin{array}{l}\text { Población } \\
\text { geoquímica }\end{array}$ & No de puntos & $\begin{array}{c}\text { Porcentaje } \\
(\%)\end{array}$ & $\begin{array}{l}\text { Media concentración } \\
\mathrm{CO}_{2}(\mathrm{ppmV})\end{array}$ & $\begin{array}{l}\text { Desviación estándar intervalo } \\
\text { de confianza del 95\%(ppmV) }\end{array}$ \\
\hline \multirow[t]{4}{*}{2000} & fondo & 88 & 79,6 & 605 & $575-641$ \\
\hline & intermedia & 17 & 16,5 & 4815 & $4055-5947$ \\
\hline & pico & 5 & 3,9 & 79821 & $72948-88841$ \\
\hline & TOTAL & 110 & 100 & & \\
\hline \multirow[t]{4}{*}{2001} & fondo & 145 & 79,9 & 408 & $399-419$ \\
\hline & intermedia & 31 & 17,3 & 6116 & $4141-10366$ \\
\hline & pico & 6 & 2,8 & 422583 & $413455-433570$ \\
\hline & TOTAL & 182 & 100 & & \\
\hline \multirow[t]{4}{*}{2002} & fondo & 169 & 69,4 & 578 & ne \\
\hline & intermedia & 46 & 18,8 & 8987 & $6995-12348$ \\
\hline & pico & 29 & 11,8 & 171393 & $136326-228638$ \\
\hline & TOTAL & 242 & 100 & & \\
\hline \multirow[t]{4}{*}{2003} & fondo & 138 & 58,0 & 388 & $380-399$ \\
\hline & intermedia & 58 & 24,7 & 2537 & $2111-3181$ \\
\hline & pico & 42 & 17,3 & 8014 & $7324-8920$ \\
\hline & TOTAL & 238 & 100 & & \\
\hline
\end{tabular}

n.e.: no es posible estimar el intervalo de confianza 
Cuadro 3

Resumen del análisis estadístico-gráfico aplicado a los datos de flujo difuso de $\mathrm{CO}_{2}$ del volcán Poás

\begin{tabular}{|c|c|c|c|c|c|}
\hline Campaña & $\begin{array}{l}\text { Población } \\
\text { geoquímica }\end{array}$ & No de puntos & $\begin{array}{c}\text { Porcentaje } \\
(\%)\end{array}$ & $\begin{array}{l}\text { Media flujo } \mathrm{CO}_{2} \\
\quad\left(\mathrm{~g} \mathrm{~m}^{-2} \mathrm{~d}^{-1}\right)\end{array}$ & $\begin{array}{c}\text { Desviación estándar intervalo } \\
\text { de confianza del } 95 \% \\
\left(\mathrm{~g} \mathrm{~m}^{-2} \mathrm{~d}^{-1}\right)\end{array}$ \\
\hline \multirow[t]{4}{*}{2000} & fondo & 79 & 61,9 & 1,3 & $1,1-1,6$ \\
\hline & intermedia & 28 & 22,6 & 12,4 & $11,2-14,1$ \\
\hline & pico & 22 & 15,5 & 354 & $242-591$ \\
\hline & TOTAL & 129 & 100 & & \\
\hline \multirow[t]{4}{*}{2001} & fondo & 165 & 91,5 & 7.5 & $6,4-9,1$ \\
\hline & intermedia & 9 & 5,1 & 275 & $258-296$ \\
\hline & pico & 7 & 3,4 & 1663 & $1651-1674$ \\
\hline & TOTAL & 181 & 100 & & \\
\hline \multirow[t]{4}{*}{2002} & fondo & 211 & 85,7 & 4.6 & $3,6-6,2$ \\
\hline & intermedia & 16 & 6,4 & 250 & $208-314$ \\
\hline & pico & 18 & 7,9 & 1996 & n.e. \\
\hline & TOTAL & 245 & 100 & & \\
\hline \multirow[t]{4}{*}{2003} & fondo & 223 & 92,7 & 14.8 & $10,1-25,2$ \\
\hline & intermedia & 10 & 4,2 & 181 & $158-213$ \\
\hline & pico & 8 & 3,1 & 4498 & $3134-7512$ \\
\hline & TOTAL & 241 & 100 & & \\
\hline
\end{tabular}

n.e.: no es posible estimar el intervalo de confianza

atmósfera, se realizaron los mapas de distribución espacial basados en la aplicación del algoritmo de simulación gaussiana (sGs) por medio del programa GSLIB (Deutsch \& Journel 1998). Este método de interpolación ha sido ampliamente utilizado en el estudio de desgasificación difusa de otros sistemas volcánicos del mundo (Cardellini et al. 2003, Chiodini et al, 2004; Frondini et al., 2004, Chiodini et al., 2007; Melián et al. 2007; Padrón et al., 2008). La aplicación del algoritmo sGs para la realización de los mapas de distribución espacial de concentración y flujo difuso de $\mathrm{CO}_{2}$ en las zonas no muestreadas nos permite evaluar la incertidumbre en el cálculo de la emisión total de $\mathrm{CO}_{2}$ a la atmósfera en el área de estudio. Basándonos en el modelo de variograma, se realizaron 100 simulaciones sobre un grid de 42752 celdas $(7 \mathrm{~m} \times 10 \mathrm{~m}$ ) para cada uno de los mapas de distribución espacial de concentración y flujo difuso de $\mathrm{CO}_{2}$. Los valores obtenidos en las 100 simulaciones fueron promediados para la construcción de los mapas de distribución espacial.
Los variogramas teóricos obtenidos en función de los variogramas experimentales fueron ajustados con un modelo esférico para la concentración y flujo de $\mathrm{CO}_{2}$ en las cuatro campañas realizadas, salvo para el estudio del flujo de $\mathrm{CO}_{2}$ en el año 2002 donde el variograma se ajustó con un modelo de efecto nugget puro.

Los mapas de distribución espacial de las anomalías de flujo difuso de $\mathrm{CO}_{2}$ muestran que en todas las campañas la mayor parte del área de estudio presentaba valores por debajo de los 10 $\mathrm{g} \mathrm{m}^{-2} \mathrm{~d}^{-1}$, y cercano al valor medio de la población de fondo (Fig. 2), donde se observan que las principales anomalías de flujo de $\mathrm{CO}_{2}$ están localizadas en el interior del cráter principal para los cuatro estudios realizados. El valor más alto de flujo de $\mathrm{CO}_{2}$ fue medido en el año 2000 y se localizó en el interior del cráter principal, al $\mathrm{SO}$ del domo, con un valor superior a los $1500 \mathrm{~g} \mathrm{~m}^{-2}$ $\mathrm{d}^{-1}$ (Fig. 2A). En el domo, situado en el interior del cráter principal, los valores de flujo de $\mathrm{CO}_{2}$ fueron superiores a los $800 \mathrm{~g} \mathrm{~m}^{-2} \mathrm{~d}^{-1}$, coincidiendo 

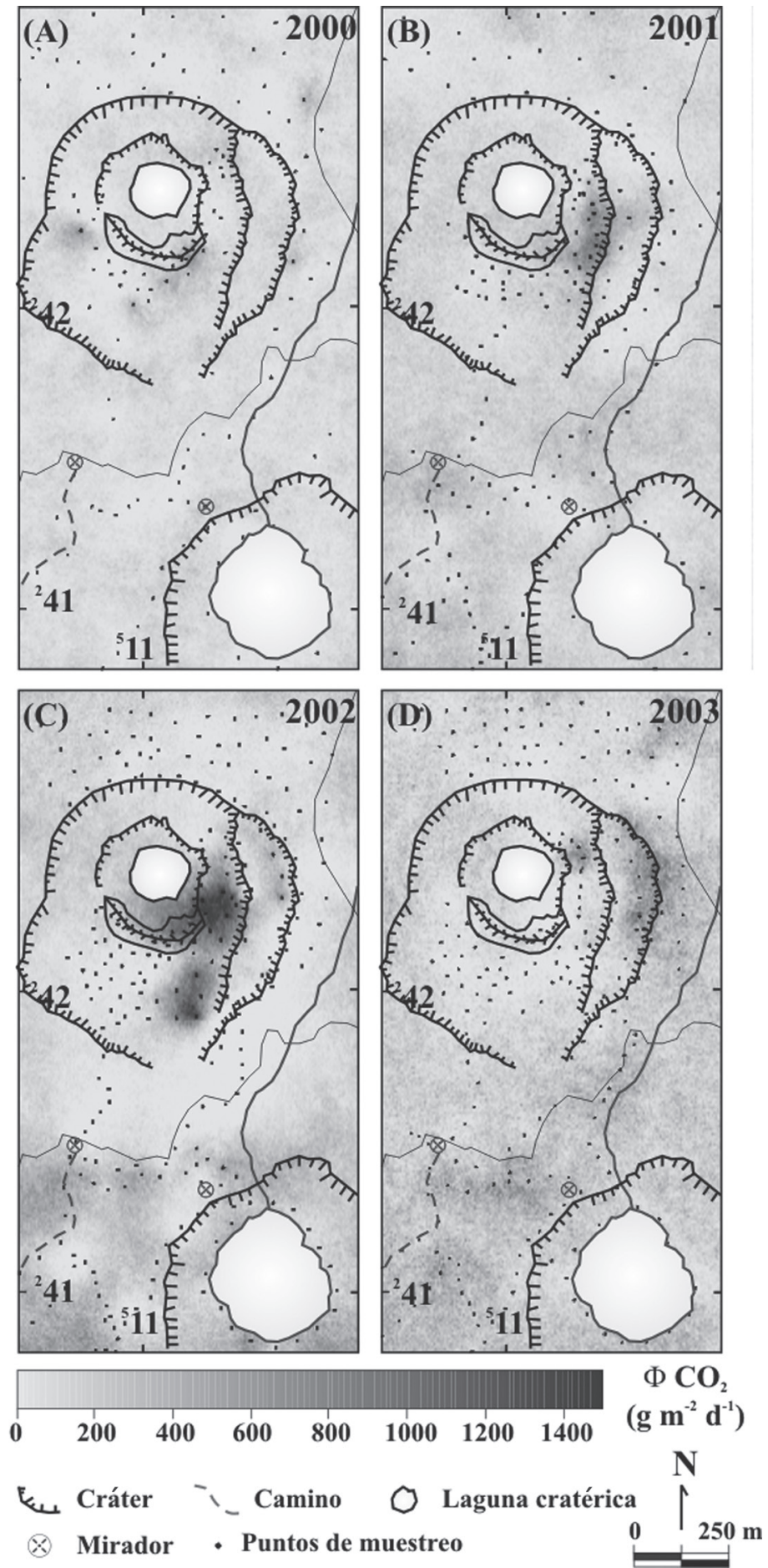

Fig. 2: Distribución espacial del flujo difuso de $\mathrm{CO}_{2}$ en el gas del suelo $\left(\mathrm{g} \mathrm{m}^{-2} \mathrm{~d}^{-1}\right)$ en el Poás, Costa Rica en: (A) abril 2000, (B) marzo 2001, (C) marzo 2002 y (D) abril 2003. Los puntos indican los lugares de muestreo. 
con la zona de actividad fumarólica más intensa durante ese año. En el interior del cráter principal se midieron también flujos de $\mathrm{CO}_{2}$ superiores a los $400 \mathrm{~g} \mathrm{~m}^{-2} \mathrm{~d}^{-1}$ al sur del domo y en el noreste del mismo. Así mismo, en el sector E del área de estudio y en el borde norte del cráter principal se midieron valores altos de flujo de $\mathrm{CO}_{2}(>600$ $\mathrm{g} \mathrm{m}^{-2} \mathrm{~d}^{-1}$ ). Vaselli et al. (2003) reportan para el periodo 1999 y 2000 un aumento en el contenido de cloruros de los condensados fumarólicos, sugiriendo la existencia de un aumento en la liberación de fluidos del sistema hidrotermal. Los cambios observados en la composición química de las fumarolas del Poás es el primer estado de los cambios que serán observados en las emisiones difusas del Poás en los años sucesivos.

La distribución espacial de los valores de flujo difuso de $\mathrm{CO}_{2}$ en la campaña de 2001 mostró la existencia de un cambio significativo en la localización de las anomalías en el interior del cráter principal, situándose los mayores valores de flujo $\left(>1000 \mathrm{~g} \mathrm{~m}^{-2} \mathrm{~d}^{-1}\right)$ en el sector este del cráter, donde se midieron valores cercanos a los $2129 \mathrm{~g} \mathrm{~m}^{-2}$ $\mathrm{d}^{-1}$ (Fig. 2B). Hay que destacar el cambio producido en los valores de flujo $\mathrm{CO}_{2}$ en el domo, donde se pasó de valores en torno a los $800 \mathrm{~g} \mathrm{~m}^{-2} \mathrm{~d}^{-1}$ en el año 2000, a valores de $500 \mathrm{~g} \mathrm{~m}^{-2} \mathrm{~d}^{-1}$. También se localizaron valores relativamente altos de flujo de $\mathrm{CO}_{2}$ en la zona sur del área de estudio donde se midieron valores superiores a los $700 \mathrm{~g} \mathrm{~m}^{-2}$ $\mathrm{d}^{-1}$. Durante este año se produjo un aumento de la temperatura del suelo en la terraza este del cráter. La concentración de $\mathrm{H}_{2}$ en el suelo durante este año aumentó de forma drástica pasando de valores máximos de 1,6 ppmV a valores de 1910 ppmV. Los niveles de sismicidad Tipo A registrados en el Poás también presentaron un aumento significativo mientras que la actividad fumarólica se incrementa en la parte NE del cráter principal. Durante el periodo 2000 y 2001, los datos gravimétricos registrados en el interior del cráter principal mostraron un incremento significativo al sur y oeste de la laguna y un decrecimiento pronunciado de los valores de microgravimetría al norte y este de la misma, mientras en el domo volcánico no se observan variaciones significativas (Rymer et al., 2005). El incremento en la emisión difusa de $\mathrm{CO}_{2}$ pudiera estar relacionada con los cambios observados en la microgravimetría. Este aumento de los niveles de gravimetría al sur y oeste del domo volcánicos son interpretados por Rymer et al. (2005) como una posible intrusión magmática en el sistema volcánico-hidrotermal del Poás.

Durante el año 2002 se observó un aumento significativo del flujo de $\mathrm{CO}_{2}$ en toda la zona de estudio y principalmente en el interior del cráter principal (Fig. 2C), pudiéndose distinguir 4 zonas con valores altos de flujo de $\mathrm{CO}_{2}$ : (1) al E, donde en 2001 se comenzó a producir un incremento del flujo de $\mathrm{CO}_{2}\left(>1500 \mathrm{~g} \mathrm{~m}^{-2} \mathrm{~d}^{-1}\right)$; (2) en el domo, donde se produjo un notable incremento del flujo de $\mathrm{CO}_{2}$ con respecto a 2000 y 2001, pasando de valores mayores de $800 \mathrm{~g} \mathrm{~m}^{-2} \mathrm{~d}^{-1}$ en 2000 y de 500 $\mathrm{g} \mathrm{m}^{-2} \mathrm{~d}^{-1}$ en 2001 a valores superiores a los $1,300 \mathrm{~g}$ $\mathrm{m}^{-2} \mathrm{~d}^{-1}$ en 2002; (3) al sur del domo cratérico donde se alcanzaron valores de hasta $1.400 \mathrm{~g} \mathrm{~m}^{-2} \mathrm{~d}^{-1} \mathrm{y}$ (4) en la terraza localizada al E del cráter, donde se incrementó notablemente el flujo de $\mathrm{CO}_{2}$ y se alcanzaron valores $>1200 \mathrm{~g} \mathrm{~m}^{-2} \mathrm{~d}^{-1}$. Al suroeste del área de estudio se midieron también valores relativamente altos de flujo de $\mathrm{CO}_{2}$, en torno a los $700 \mathrm{~g} \mathrm{~m}^{-2} \mathrm{~d}^{-1}$, así como en el borde sur de la laguna Botos $>900 \mathrm{~g} \mathrm{~m}^{-2} \mathrm{~d}^{-1}$. Durante este mismo año, continuó el aumento en la extensión de las anomalías de concentración de $\mathrm{H}_{2}$ y temperatura del suelo dentro del cráter principal y en la terraza este del cráter (Melián et al., 2007).

La distribución de anomalías de flujo de $\mathrm{CO}_{2}$ para el año 2003 mostró un claro cambio en la localización de los mayores valores de flujo de $\mathrm{CO}_{2}$ (Fig. 2D). Se observó una notable disminución de dichos valores de flujo con respecto a los medidos en el año 2002, aunque es en este año cuando se midieron los valores más altos de flujo de $\mathrm{CO}_{2}$ de todo el estudio $\left(4.712 \mathrm{~g} \mathrm{~m}^{-2} \mathrm{~d}^{-1}\right)$. Las principales anomalías de flujo de $\mathrm{CO}_{2}$ se distribuyeron en tres zonas dentro del cráter principal: (1) en el sector noreste y este del cráter con valores de flujo de $\mathrm{CO}_{2}$ superiores a los $1.500 \mathrm{~g} \mathrm{~m}^{-2} \mathrm{~d}^{-1}$, estos valores disminuyeron considerablemente con respecto a los obtenidos en el año 2002, (2) en la terraza este con valores de flujo de $\mathrm{CO}_{2}>1.000 \mathrm{~g} \mathrm{~m}^{-2} \mathrm{~d}^{-1}$ y (3) en el domo, aunque hay que destacar la considerable disminución de los valores de flujo difuso de $\mathrm{CO}_{2}$ en el domo ya que en este año se midieron los flujos más bajos de todo el estudio $\left(300 \mathrm{~g} \mathrm{~m}^{-2} \mathrm{~d}^{-1}\right)$. A lo largo del borde noreste 

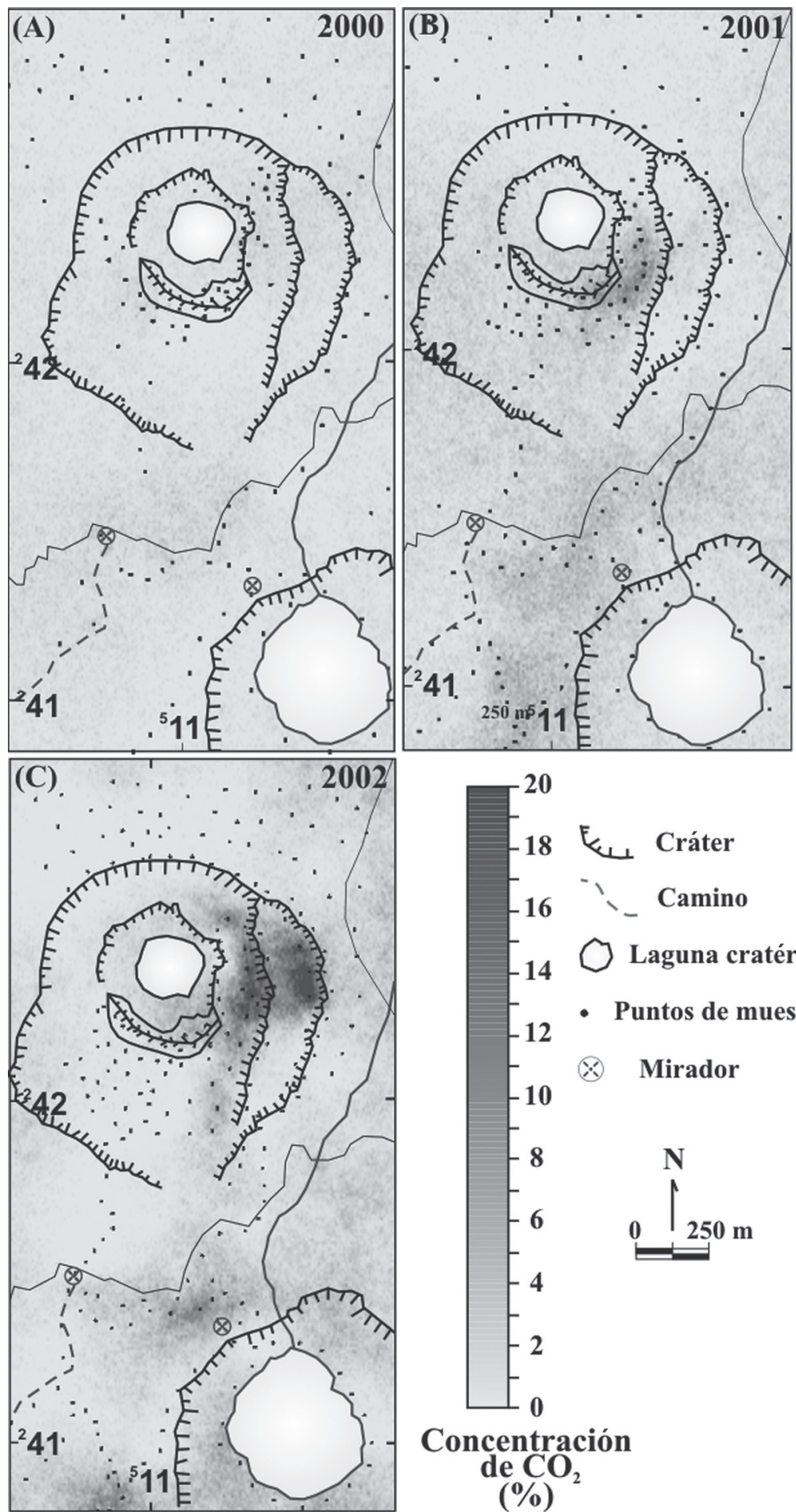

Fig. 3: Distribución espacial de la concentración de $\mathrm{CO}_{2}$ en el gas del suelo (\%V) en el Poás, Costa Rica en: (A) abril 2000, (B) marzo 2001 y (C) marzo 2002. Los puntos indican los lugares de muestreo. 
del cráter principal se midieron también valores altos de flujo de $\mathrm{CO}_{2}\left(>1100 \mathrm{~g} \mathrm{~m}^{-2} \mathrm{~d}^{-1}\right)$. Durante este año, Melián et al. (2007), reportan una disminución de los valores de concentración de $\mathrm{H}_{2}$ al igual que se observa con la concentración de $\mathrm{CO}_{2}$ en el suelo probablemente debido a que el sistema ya se ha desgasificado, lo que favorece que los gases y volátiles presentes en el sistema prefieran el escape a través de las ventanas de desgasificación abiertas.

En los mapas de distribución espacial de la concentración de $\mathrm{CO}_{2}$ se observa que la mayor parte del área de estudio presentó valores de concentración de $\mathrm{CO}_{2}$ en torno a los 1000 ppm, valor 3 veces superior a la concentración de $\mathrm{CO}_{2}$ en el aire y próximo al valor de fondo estimado para cada una de las campañas (entre 400 y 600 ppm). En todas las campañas realizadas en el Poás durante el periodo de estudio, se observaron dos zonas anómalas de $\mathrm{CO}_{2}$; (1) dentro del cráter principal, donde se midieron los valores más altos de concentración de $\mathrm{CO}_{2}$ y (2) en la zona sur del área de estudio y a lo largo del camino que lleva al cráter principal y donde existe una gran cantidad de vegetación. El mapa de distribución espacial para el año 2000 muestra que las anomalías de concentración de $\mathrm{CO}_{2}$ en el suelo del Poás están localizadas en la zona del camino que lleva al mirador del cráter principal, con valores máximos de hasta un $5,0 \% \mathrm{~V}$ de $\mathrm{CO}_{2}$ (Fig. 3A).

El mapa de distribución espacial de las anomalías de concentración de $\mathrm{CO}_{2}$ para la campaña de 2001 muestra un aumento general de estos valores en toda el área de estudio respecto al año 2000 (Fig. 3B). En la zona sur del área de estudio se observó un claro aumento tanto de los valores anómalos de concentración de $\mathrm{CO}_{2}$ como de la extensión del área afectada por valores anómalos y cercanos al $8 \% \mathrm{~V}$. Dentro del cráter principal, se pasó de medir valores puntuales de concentraciones altas de $\mathrm{CO}_{2}$ a una extensa área afectada por valores de concentración de $\mathrm{CO}_{2}$ superiores al $18 \% \mathrm{~V}$, llegando incluso a alcanzarse valores puntuales superiores al $50 \% \mathrm{~V}$ de $\mathrm{CO}_{2}$ al noreste del cráter principal. Esta área con valores altos de $\mathrm{CO}_{2}$ se extiende hasta el domo cratérico donde se pasó de valores en torno a $1,0 \% \mathrm{~V}$ de $\mathrm{CO}_{2}$ medidos en la campaña del 2000 a valores de concentración de $\mathrm{CO}_{2}$ superiores a un $14 \% \mathrm{~V}$. Otras zonas que presentaron valores anómalos de concentración de $\mathrm{CO}_{2}$ se localizaron al sur del domo (concentración de $\mathrm{CO}_{2}>10 \% \mathrm{~V}$ ).

En la campaña realizada en el año 2002 se midieron los mayores valores de concentración de $\mathrm{CO}_{2}$ en el ambiente superficial del Poás durante el período de estudio (Fig. 3C). El interior del cráter principal presentó una amplia zona con valores altos de concentración de $\mathrm{CO}_{2}(>20 \% \mathrm{~V})$, localizados en la pared este del cráter principal, donde pudieron medirse valores puntuales superiores al $50 \% \mathrm{~V} \mathrm{CO}_{2}$. También se midieron valores altos de concentración de $\mathrm{CO}_{2}(>8 \% \mathrm{~V}$ de $\mathrm{CO}_{2}$ ) en el domo y al sureste del mismo, y en el interior del cráter principal. En la terraza localizada al este del cráter principal se observó un amplio sector con valores altos de concentración de $\mathrm{CO}_{2}(>22 \% \mathrm{~V})$, en este sector se observaron valores puntuales superiores al $50 \% \mathrm{~V}$ de $\mathrm{CO}_{2}$ $(76,8 \% \mathrm{~V})$. Es en esta zona donde se midieron los mayores valores de concentración de $\mathrm{CO}_{2}$ durante la campaña de 2002. Cabe reseñar que esta zona anómala no existía en los años anteriores. Al sur del área de estudio y en el borde del camino que lleva al mirador del cráter principal se midieron de nuevo valores altos de concentración $\mathrm{CO}_{2}$ con un mayor número de anomalías puntuales respecto a 2001. Otra zona que presentó valores altos de concentración de $\mathrm{CO}_{2}$ se localizó al sureste de la laguna Botos con valores de hasta un $6 \% \mathrm{~V}$ de $\mathrm{CO}_{2}$.

En el 2003, las anomalías de concentración de $\mathrm{CO}_{2}$ disminuyeron respecto a los años 2001 y 2002 , aunque las zonas que presentaron los valores más altos de concentración de $\mathrm{CO}_{2}$ coincidieron con las de años anteriores (Fig. 4). La zona sur del área de estudio presentó valores de concentración de $\mathrm{CO}_{2}$ superiores al $1,2 \% \mathrm{~V}$, aunque fueron menores a los registrados en años anteriores. Dentro del cráter principal se observó una importante disminución de los valores de concentración de $\mathrm{CO}_{2}$. La concentración de $\mathrm{CO}_{2}$ en el domo cratérico continuó disminuyendo, pasando de valores máximos medidos de $14 \% \mathrm{~V}$ en 2001 a un $8 \% \mathrm{~V}$ en 2002 y a valores de fondo $>0,07 \% \mathrm{~V}$ en 2003. En la pared este del cráter principal se observó una disminución más drástica de la concentración de $\mathrm{CO}_{2}$, pasando de valores superiores a $50 \% \mathrm{~V}$ de $\mathrm{CO}_{2}$ a valores máximos de 

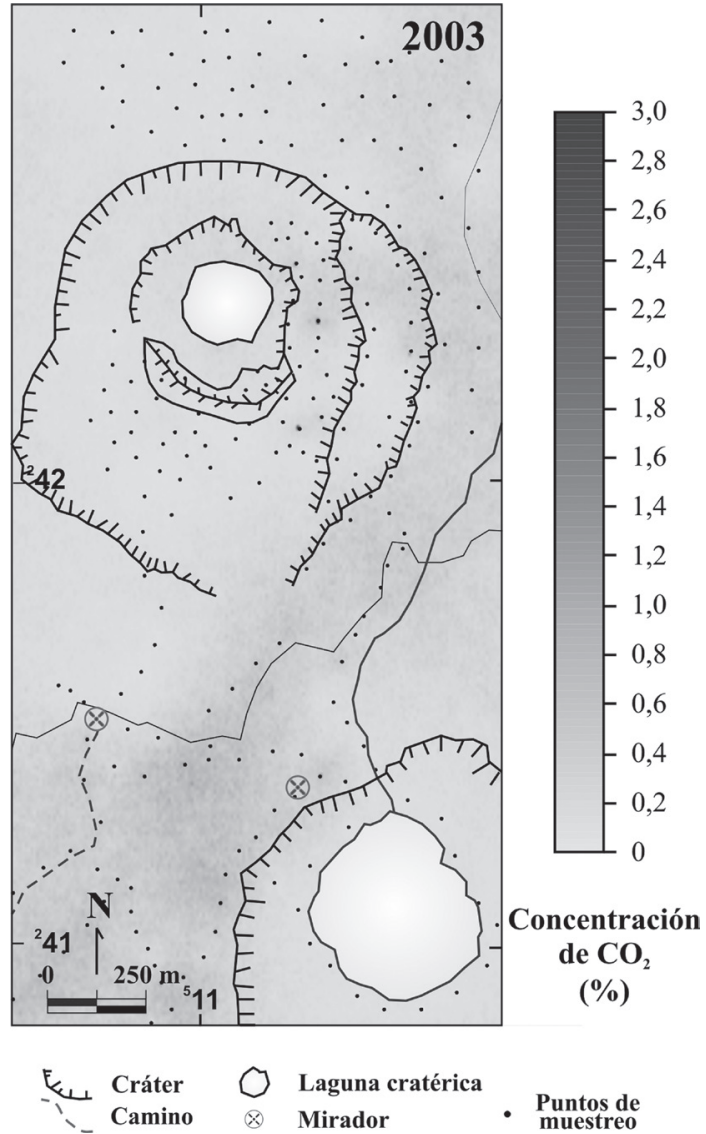

Fig. 4: Distribución espacial de la concentración de $\mathrm{CO}_{2}$ en el gas del suelo $(\% \mathrm{~V})$ en el Poás, Costa Rica en abril de 2003.

Los puntos indican los lugares de muestreo.

$4,5 \% \mathrm{~V}$. También en la terraza este del cráter principal fue evidente la disminución de la concentración de $\mathrm{CO}_{2}$ con respecto a 2002 ya que se pasó de los valores más altos medidos durante todo el estudio $(>76,8 \% \mathrm{~V})$ a valores de concentración de $\mathrm{CO}_{2}$ en torno al $1,0 \% \mathrm{~V}$.

Del total de muestras recolectadas para el análisis isotópico del gas del suelo, se seleccionaron algunas muestras representativas de todo el rango de valores de flujo y concentración de $\mathrm{CO}_{2}$ medidos en cada una de las campañas. En la campaña del 2000 se analizó el $29,5 \% \mathrm{~V}$ del total de las muestras, con valores de $\delta^{13} \mathrm{C}-\mathrm{CO}_{2}$ que variaron entre $-66,5$ y $0,9 \%$. Respecto a los años 2001 y 2002 , se analizaron el $18,8 \% \mathrm{~V}$ y el $23,2 \% \mathrm{~V}$ del total de las muestras con valores de $\delta^{13} \mathrm{C}-\mathrm{CO}_{2}$ entre $-52,8$ y $-3,97 \%$ y $-50,9$ y $0,7 \%$, respectivamente. Durante la campaña de 2003 no fue posible realizar los análisis isotópicos. En la Figura 5 se presenta la composición isotópica de $\mathrm{CO}_{2}$ en las muestras del gas del suelo para los estudios de 2000, 2001 y 2002. La mayor parte de los datos presentan valores de $\delta^{13} \mathrm{C}-\mathrm{CO}_{2}<-25 \%$, localizándose los mayores valores de $\delta^{13} \mathrm{C}-\mathrm{CO}_{2}$ durante las tres campañas en el interior del cráter principal $\left(\delta^{13} \mathrm{C}-\mathrm{CO}_{2}>-4 \%\right.$ ) con un claro incremento en el número de muestras con valores pesados de $\delta^{13} \mathrm{C}$ $\mathrm{CO}_{2}$ entren 2000 y 2002.

La Figura 6 muestra los diagramas de correlación entre el contenido de $\mathrm{CO}_{2}(\% \mathrm{~V})$ y los valores de $\delta{ }^{13} \mathrm{C}-\mathrm{CO}_{2}$ en las muestras del gas del suelo para los estudios de 2000, 2001 y 2002. En el diagrama también se presentan las líneas de mezcla entre los distintos reservorios (1) aire atmosférico $\left(\mathrm{CO}_{2}=0,036 \% \mathrm{~V}\right.$ y $\delta^{13} \mathrm{C}\left(\mathrm{CO}_{2}\right)=-8 \%$ ), (2) gas magmático $\left(\mathrm{CO}_{2}=100 \% \mathrm{~V}\right.$ y $\delta^{13} \mathrm{C}\left(\mathrm{CO}_{2}\right)$ $=-6 \%$ ) y (3) gas biogénico construido en base a los valores medios y desviaciones estándares de la población de fondo de la concentración de $\mathrm{CO}_{2}$ estimado para cada uno de los estudios y sus respectivos valores de $\delta{ }^{13} \mathrm{C}-\mathrm{CO}_{2}$. En el diagrama también se representa la composición química representativa del gas fumarólico del Poás y las correspondientes líneas de mezcla (Poás 2000: $\mathrm{CO}_{2}=11,96 \% \mathrm{~V}$ y $\delta^{13} \mathrm{C}-\mathrm{CO}_{2}=-5,75 \%$, Poás 2001: $\mathrm{CO}_{2}=54,82 \% \mathrm{~V}$ y $\delta^{13} \mathrm{C}-\mathrm{CO}_{2}=-4,46 \%$ y Poás 2002: $\mathrm{CO}_{2}=17,60 \% \mathrm{~V}$ y $\delta^{13} \mathrm{C}-\mathrm{CO}_{2}=-3,62 \%$ o (Melián, 2008).

En este diagrama puede observarse que la mayor parte de las muestras se localizan entre los reservorios volcánico y biogénico, lo que confirma el proceso de mezcla entre ambos, descartándose una perturbación atmosférica para aquellas muestras que presentan una firma isotópica de $\mathrm{CO}_{2}$ relativamente más pesada. En el 2001 y 2002 existe una mayor dispersión de los valores a lo largo de la línea de mezcla entre el gas biogénico y el reservorio volcánico-fumarólico, con un claro incremento desde 2000 a 2002. Hay que tener en cuenta que los valores de $\delta^{13} \mathrm{C}-\mathrm{CO}_{2}$ son utilizados para distinguir los 

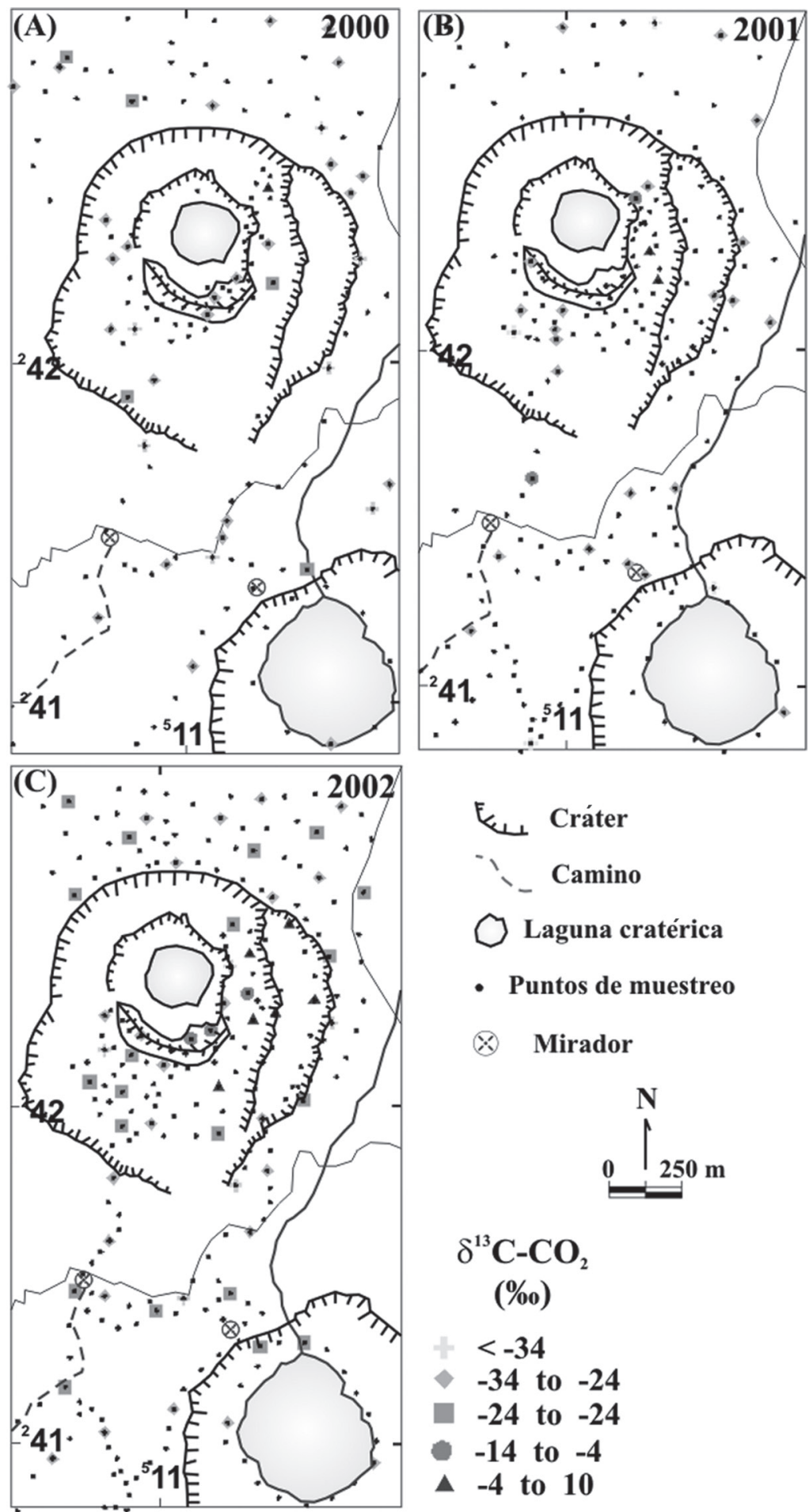

- Puntos de muestreo

Q Mirador

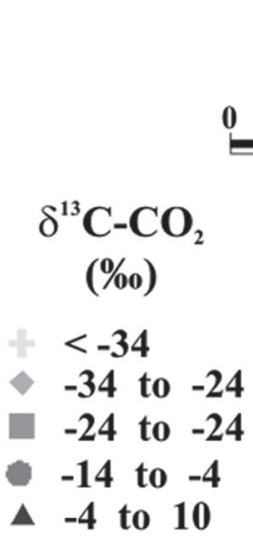

Fig. 5: Distribución espacial de la composición isotópica $\delta^{13} \mathrm{C}_{-}-\mathrm{CO}_{2}$ (\%) en el Poás, Costa Rica en: (A) abril 2000, (B) marzo 2001 y (C) marzo 2002. Los puntos indican los lugares de muestreo. 

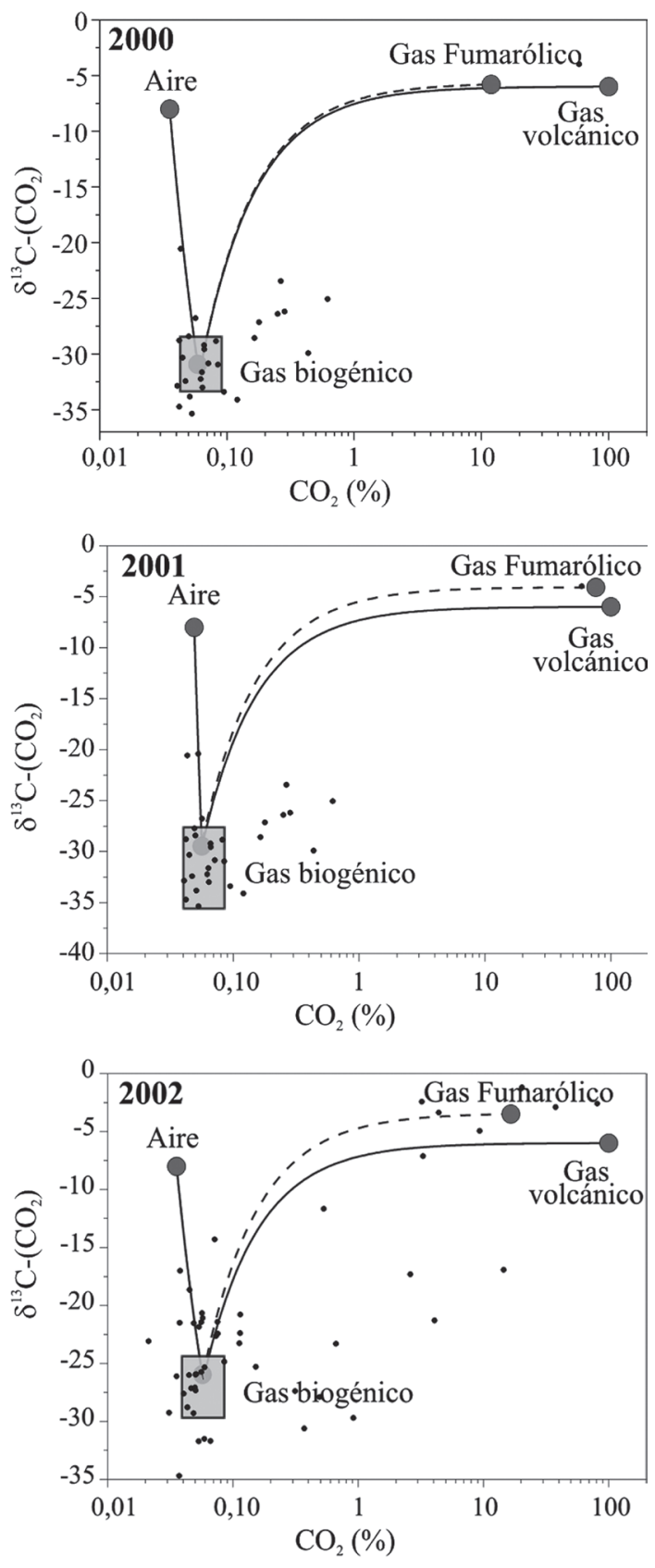

Fig. 6: Diagrama de correlación entre los valores $\delta^{13} \mathrm{C}\left(\mathrm{CO}_{2}\right) \mathrm{y}$ concentración de $\mathrm{CO}_{2}(\% \mathrm{~V})$ en la atmósfera del suelo durante las campañas (a) 2000, (b) 2001 y (c) 2002. Las líneas representan las líneas de mezcla entre los diferentes reservorios geoquímicos aire, gas magmático y gas biogénico. diferentes orígenes del $\mathrm{CO}_{2}$ en las muestras de gas (Schwarcz, 1969; Hoefs, 1980).

Los máximos valores medidos de flujo difuso de $\mathrm{CO}_{2}$ se localizaron dentro del cráter principal durante los cuatro estudios realizados, en zonas donde las manifestaciones geotermales en superficie eran obvias, es decir, junto a los conductos de emisión y donde se localizaban las emisiones visibles, destacando las anomalías localizadas en el domo para todo el período de estudio y en la pared este del mismo durante los años 2001, 2002 y 2003. En 2002 y 2003 se observaron también valores altos de flujo difuso de $\mathrm{CO}_{2}$ en la terraza E del cráter. La correlación encontrada entre la concentración y el flujo difuso de $\mathrm{CO}_{2}$ es baja en los cuatro estudios con valores de $\mathrm{r}^{2}=-0,032$ en 2000 , incrementándose en 2001 y $2002\left(\mathrm{r}^{2}=0,55\right.$ y 0,52 respectivamente) y decreciendo nuevamente en 2003 hasta $r^{2}=0,0022$. Esta baja correlación probablemente es debida a la gran cantidad de agua existente en los suelos del Poás. El alto contenido en agua del suelo, puede disminuir el flujo de $\mathrm{CO}_{2}$ debido a que al ser una especie ácida, parte del mismo se queda retenido en el agua. Las relaciones isotópicas $\delta^{13} \mathrm{C}-\mathrm{CO}_{2}$ medidas en los gases procedentes del suelo muestran una relativa buena correlación con los valores de flujo difuso de $\mathrm{CO}_{2}(0,013 ; 0,55$ y 0,62 para 2000,2001 y 2002 , respectivamente). Esta correlación positiva se observa también en los valores de temperatura del suelo y concentración de $\mathrm{H}_{2}$, vapor de $\mathrm{Hg}^{0}$ y $\mathrm{H}_{2} \mathrm{~S}$ (Melián et al., 2002, 2003, 2007; Nolasco et al., 2003; Melián, 2008). La correlación positiva encontrada entre el flujo de $\mathrm{CO}_{2}$, las relaciones isotópicas $\delta^{13} \mathrm{C}_{-} \mathrm{CO}_{2}$, la temperatura del suelo y las concentraciones de $\mathrm{H}_{2}$, vapor de $\mathrm{Hg}^{0}$ y $\mathrm{H}_{2} \mathrm{~S}$ nos indica una importante contribución magmática a estas emisiones. Los resultados obtenidos en el estudio de la composición isotópica de las fumarolas del Poás $\left({ }^{3} \mathrm{He} /{ }^{4} \mathrm{He}\right.$, y $\left.\delta^{13} \mathrm{C}\right)$ también nos indican un aporte de profundidad de las emisiones volcánicas del Poás (Melián, 2008).

A partir de los mapas de distribución espacial de flujo difuso de $\mathrm{CO}_{2}$, se ha estimado la tasa de emisión de $\mathrm{CO}_{2}$ a la atmósfera por el Poás en cada uno de los estudios realizados. Este valor de 
la tasa de emisión de $\mathrm{CO}_{2}$ se obtiene a partir de promediar los valores de emisión de las 100 simulaciones realizadas en cada estudio. El valor de la incertidumbre vendrá dado por la desviación estándar del flujo calculado para las 100 simulaciones realizadas. La estimación se realiza multiplicando el valor medio del flujo difuso de $\mathrm{CO}_{2}$ en cada celda por el área de celda. La suma de todas las celdas nos proporciona el valor del flujo total emitido en cada estudio. La estimación de la emisión total de $\mathrm{CO}_{2}$ en forma difusa a la atmósfera por el Poás para el área de estudio $\left(3,2 \mathrm{~km}^{2}\right)$ es de $164 \pm 15 \mathrm{t} \mathrm{d}^{-1}$ para el año 2000, en el 2001 este valor aumentó hasta $423 \pm 54 \mathrm{t} \mathrm{d}^{-1}$, mientras que para el 2002 y 2003 este valor fue de $537 \pm 69 \mathrm{t}$ $\mathrm{d}^{-1}$ y $542 \pm 63 \mathrm{t} \mathrm{d}^{-1}$, respectivamente (Melián et al., 2006, Melián, 2008).

Con el fin de relacionar las variaciones temporales de la emisión difusa de $\mathrm{CO}_{2}$ con la actividad volcánica del Poás, hemos utilizado el concepto de "aceleración del proceso de desgasificación difusa de $\mathrm{CO}_{2}$ ". La "aceleración" se calcula dividiendo el cambio en la tasa total de emisión de $\mathrm{CO}_{2}$ ( $\mathrm{t}$ d-1) entre el período de tiempo trascurrido (d), obteniéndose unidades en $\mathrm{t} \mathrm{d}^{-2}$. Esta "aceleración" nos informa de la dinámica y/o el ritmo con el aumenta o disminuye del proceso de desgadificación. Durante el periodo de estudio, los valores de aceleración no se corresponden con los de las tasas de emisión. Así, el mayor valor de aceleración $\left(0,94 \mathrm{t} \mathrm{d}^{-2}\right)$ correspondió al periodo $2000 \mathrm{y}$ 2001, siendo en 2002 cuando se midió la mayor tasa de emisión de $\mathrm{CO}_{2}$ pero sin embargo una aceleración del sistema de $0,35 \mathrm{t} \mathrm{d}^{-2}$. Lo mismo se ha observado para el año 2003, con una disminución notable de la aceleración hasta $0,014 \mathrm{t} \mathrm{d}^{-2}$. Este comportamiento de la emisión difusa de $\mathrm{CO}_{2}$ en el Poás sigue el modelo propuesto por Notsu et al. (2006), donde los cambios en la actividad volcánica producidos por inyección de nuevo material más gasificado generan en un primer estadio un aumento en la emisión difusa de $\mathrm{CO}_{2}$. En un segundo estadio, se observa un incremento tanto en la tasa de emisión difusa, como en la visible. Estos niveles de emisión difusa permanecen relativamente constantes (2001-2003) hasta que se produzca una erupción con la consecuente liberación de la energía del sistema.

En marzo del 2001 y 2003, Zimmer et al. (2004), realizaron un estudio de la emisión de $\mathrm{SO}_{2}$ en el Poás mediante el uso de sensores ópticos remotos como el COSPEC. En este estudio se estimó para 2001 una tasa de emisión de $\mathrm{SO}_{2}$ de $8,30 \pm 4,00 \mathrm{t} \mathrm{d}^{-1}\left(1,30 \bullet 10^{5} \pm 6,25 \cdot 10^{4} \mathrm{~mol}\right.$ $\left.\mathrm{d}^{-1}\right)$, mientras que en 2003 fue de 29,0 $\pm 9,00 \mathrm{t}$ $\mathrm{d}^{-1}\left(4,53 \cdot 10^{5} \pm 1,41 \cdot 10^{4} \mathrm{~mol} \mathrm{~d}^{-1}\right)$. Los valores de flujo de $\mathrm{SO}_{2}$ en 2001 y 2003 fueron inferiores a los medidos entre 1981 y 1983 por Andres \& Kasgnoc (1998), quienes estimaron una emisión total de $\mathrm{SO}_{2}$ en torno a los $5,0 \cdot 10^{5} \mathrm{~kg} \mathrm{~d}^{-1}$. Zimmer et al. (2004), atribuyen este valor tan bajo de emisión de $\mathrm{SO}_{2}$ a las diferencias en el grado de actividad del sistema en los diferentes años de estudio. A partir de estos datos Zimmer et al. (2004) estimaron los valores de flujo total de $\mathrm{CO}_{2}$ en función de los datos de flujo de $\mathrm{SO}_{2} \mathrm{y}$ las concentraciones de los gases medidas en las fumarolas. Ellos estiman un flujo total de $\mathrm{CO}_{2}$ en las emisiones visibles de $49 \mathrm{t} \mathrm{d}^{-1}$ para $2001 \mathrm{y}$ de 47,2 $\mathrm{t} \mathrm{d}^{-1}$ para 2003. Los valores obtenidos de flujo de $\mathrm{CO}_{2}$ mediante el estudio de desgasificación difusa fueron superiores a los obtenidos en las emisiones visibles del Poás $\left(423 \pm 54 \mathrm{t} \mathrm{d}^{-1} \mathrm{y}\right.$ $542 \pm 63 \mathrm{t} \mathrm{d}^{-1}$ para 2001 y 2003 , respectivamente). En otros sistemas volcánicos como Masaya, Nicaragua (Pérez et al., 2000), Vulcano, Italia (Baubron et al., 1990; Chiodini et al., 1998; Italiano et al., 1998) o Etna, Italia, (Allard et al., 1991) se han realizado comparaciones entre las emisiones visibles y difusas de $\mathrm{CO}_{2}$. Pérez et al. (2000) encontró que la relación desgasificación difusa/pluma para las emisiones de $\mathrm{CO}_{2}$ en el volcán Masaya, Nicaragua, es del orden de 9. El estudio realizado en Vulcano, Italia, puso de manifiesto la importante cantidad de $\mathrm{CO}_{2}$ emitida por este sistema volcánico de forma difusa y que se estima tres veces superior a la emisión del cráter en forma visible (Baubron et al., 1990; Chiodini et al., 1998; Italiano et al., 1998). La presencia de la laguna cratérica en el Poás disminuye de forma importante las emisiones visibles en el Poás. Una parte importante de los 
gases ácidos como el $\mathrm{CO}_{2}$ que son emitidos por las fumarolas presentes en el fondo de la laguna quedan disueltos en la misma, lo explica que los valores de flujo de $\mathrm{CO}_{2}$ mediante el estudio de desgasificación difusa sean superiores a los obtenidos en las emisiones visibles.

Debido a que los procesos en profundidad son complejos y no se desarrollan de forma independiente, el incremento de los valores de microgravimetría registrados en el volcán Poás en la zona sur-oeste de la laguna del cráter principal pueden estar relacionados con la sismicidad anómala presente en el volcán a finales de 1999 y los picos de actividad sísmica Tipo A registrados en el Poás durante el período de estudio. La inestabilidad del sistema ocasionada por la actividad sísmica que comienza a mediados de 1999 (Mora et al., 2004), pudo generar un cambio en la presión interna de la cámara magmática del Poás dando lugar a la generación de una pequeña intrusión dendrítica en la zona oeste de la laguna (Rymer et al., 2005). La formación de la intrusión dendrítica favorecería el drenaje de volátiles en el sector E del cráter debido a que la incorporación de una masa intrusiva en el sector oeste puede generar la formación de fracturas circulares y un incremento en la permeabilidad vertical. La fuerte emisión presentada por las fumarolas del sector E del cráter a partir de 2000 puede explicarse debido a un exceso de agua en el sistema (Vaselli et al., 2003). Esta sobrecarga de agua puede haber cambiado las condiciones redox del sistema en profundidad de P-T en el sistema lo que se refleja en las emisiones de gases y volátiles del Poás.

Los cambios observados tanto en la composición química de los gases fumarólicos del Poás (Vaselli et al., 2003; Zimmer et al., 2004) como en la sismicidad (Mora et al., 2004) y microgravimetría (Rymer et al., 2000; 2005; Locker et al., 2003; Fournier et al., 2004), así como las variaciones observadas en los valores medidos de concentración y flujo difuso de $\mathrm{CO}_{2}$ y concentración de $\mathrm{H}_{2}$ (Melián et al., 2007), corroboran la hipótesis de una pequeña intrusión dendrítica en la zona oeste de la laguna del Poás.

\section{CONCLUSIONES}

El estudio de flujo difuso y concentración de $\mathrm{CO}_{2}$ realizado en el Poás durante el período 2000-2003 muestra la existencia de una estrecha relación entre los cambios observados en la emisión de gases y la actividad volcánica del Poás. Durante este estudio, las principales anomalías espaciales del flujo y concentración de $\mathrm{CO}_{2}$ se localizaron alrededor de las zonas afectadas por actividad fumarólica, donde el suelo está más fracturado y la permeabilidad vertical es mayor. La evolución temporal del flujo difuso y concentración de $\mathrm{CO}_{2}$ indica la existencia de un aumento significativo en la emisión de gases de origen profundo durante el año 2002. Este pulso en la emisión de gases parece estar relacionado con un cambio en las condiciones de presión y/o temperatura del sistema volcánico-hidrotermal, reflejándose también en un aumento en la actividad fumarólica en la pared E del cráter (Melián et al., 2001; Vaselli et al., 2003) y cambios de microgravimetría debidos a una intrusión dendrítica producida en la zona oeste de la laguna (Rymer et al., 2005). Los bajos valores de emisión difusa de $\mathrm{CO}_{2}$ medidos en el año 2000 pudieran estar debidos a que la liberación de gases y volátiles durante el primer estado de la intrusión se realizó preferentemente a través de las principales fracturas y el conductos principal del Poás, favoreciendo la emisión visible (fumarolas y pluma volcánica) versus la emisión difusa. Estos resultados sugieren que los cambios observados tanto en las emisión difusa como en la emisión visible y las variaciones tanto en la sismicidad como en la gravimetría han sido precursores de la secuencia eruptiva ocurrida en el Poás entre MarzoOctubre de 2006.

La continuación de los estudios de desgasificación difusa de gases y volátiles conjuntamente con los estudios de gravimetría y sismicidad en el volcán Poás nos permitirían entender mejor los procesos que ocurren en sistema volcánico-hidrotermal del Poás. 


\section{AGRADECIMIENTOS}

Agradecemos a Don Juan Dobles y a todos los guardaparques del Parque Nacional Volcán Poá su hospitalidad y amabilidad durante nuestra estancia en las instalaciones del Parque Nacional del Poás. También queremos agradecer al grupo COVIRENAS, a su director Carlos Cordero, a los estudiantes de la Escuela Centroamericana de Geología, Universidad de Costa Rica y a Juan Carlos Mesa por su ayuda desinteresada en las labores de campo. Este trabajo se ha podido realizar gracias a la financiación por parte del Cabildo Insular de Tenerife, CajaCanarias y al proyecto CGL2005-07509/ CLI del Ministerio de Ciencia y Educación del Gobierno de España.

\section{REFERENCIAS}

ALLARD, P., LE BRONEC, J., MOREL, P., VAVASSEUR, P., FAIVRE-PIERRET, R., ROBE, M., ROUSSEL, C. \& ZETWOOG, P., 1987: Geochemistry of soil gas emanations from Mt. Etna, Sicily.- Terra Cognita, 7(G17-52): 407.

ALLARD, P., CARBONELLE, J., DAJLEVIC, D., LE BRONNEC, J., MOREL, P., ROBE, M., MAURENAS, J., FAIVE-PIERRET, R., MARTIN, D., SABROUX, J., \& ZETTWOOG, P., 1991: Eruptive and diffuse emissions of CO2 from Mount Etna.- Nature, 351: 387-391.

ANDRES, R. \& KASGNOC, A., 1998: A timeaveraged inventory of subaerial volcanic sulphur emissions.- J. Geophys. Res., 103(25): 25251-25261.

BAUBRON, J., ALLARD, P. \& TOUTAIN, J., 1990: Diffuse volcanic emissions of carbon dioxide from Vulcano Island, Italia. Nature, 344: 51-53.
BAUBRON, J., MATHIEU, R. \& MIELE, G., 1991: Measurement of gas flow from soils in volcanic areas: the accumulation method. Napoli'91 International Conference on active volcanoes and Risk Mitigation.[Abstract]. Napoli, Italia, 27 Augosto-1 Septiembre: 234.

BROWN, G., DOWDEN, J., KAPADIA, P., STEVENSON, D., BARQUERO, J. \& MORALES, L., 1989: Energy budget analysis for Poás Crater Lake: Implications for predicting volcanic activity.- Nature, 339: 370-373.

CARAPEZZA, M., INGUAGGIATO, S., BRUSCA, L. \& LONGO, M., 2004: Geochemical precursors of the activity of an open-conduit volcano: The Stromboli 2002-2003 eruptive events.Geophys. Res. Lett. 31: L07620. DOI: 10.1029/2004GL019614.

CARBONELLE,J.,DAJLEVIC,D.,LEBRONCE, J., MOREL, P., OBERT, J. \& ZETTWOOG, P., 1985: Etna: Composantes sommitales et pariétales des émission de gaz carbonique.Bull. PIRPSEV-CNRS, 108: 1-64.

CARDELLINI, C., CHIODINI, G. \& FRONDINI, F., 2003: Application of stochastic simulation to $\mathrm{CO}_{2}$ flux from soil: Mapping and quantification of gas release.- $\mathrm{J}$. Geophys. Res., 108(B9): 2425-2438. DOI: 10.1029/202JB002165.

CASADEVALL, T., ROSE, W., GERLACH, T., GREENLAND, L., EWERT, J., WUNDERMAN, R. \& SYMONDS, R., 1983: Gas emissions and the eruptions of Mount St. Helens through 1982.- Science, 221: 1383-1385.

CASADEVALL, T., DOUKAS, M., NEAL, C., MCGIMSEY, R. \& GARDNER, C., 1994: 
Emission rates of sulphur dioxide and carbon dioxide from Redoubt Volcano, Alaska during the 1989-1990 eruptions.- J. Volcanol. Geotherm. Res., 62: 519-530.

CASERTANO, L., BORGIA, A., CIGOLINI, C., MORALES, L., MONTERO, W., GÓMEZ, M.\&FERNÁNDEZ, J., 1985: Investigaciones geofísicas y características geoquímicas de las aguas hidrotermales: Volcán Poás, Costa Rica.- Geofísica Int. 24(2): 315-332.

CASERTANO, L., BORGIA, A., CIGOLINI, C., MORALES, L., GOMEZ, M. \& FERNÁNDEZ, J., 1987: An integrated dynamic model for the volcanic activity at Poás Volcano, Costa Rica.- Bull. Volcanol. 49: 588-598.

CHIODINI, G., FRONDINI, F. \& RACO, B., 1996: Diffuse emission of $\mathrm{CO} 2$ from the Fossa crater, Vulcano Island (Italy).- Bull. Vulcanoll. 58: 41-50.

CHIODINI, G., CIONI, R., GUIDI, M., RACO, B. \& MARINI, M., 1998: Soil Flux measurements in volcanic and geothermal areas.Appl. Geochem. 13(5): 543-552.

CHIODINI, G. \& FRONDINI, F., 2001: Carbon dioxide degassing from the Albani Hills volcanic region, Cental Italy.- Chemical Geology, 177: 67-83.

CHIODINI, G., FRONDINI, F., CARDELLINI, C., GRANIREI, D., MARINI, M. \& VENTURA, G. 2001: $\mathrm{CO}_{2}$ degassing and energy release at Solfatara volcano, Campi Flegrei, Italy.- J. Geophys. Res. 106(B8): 16213-16221.

CHIODINI, G., AVINO, R., BROMBACH, T., CALIRO, S., CARDELLINI, C., DE VITA, S., FRONDINI, F., GRANIREI, D., MAROTTA, E. \& VENTURA, G., 2004: Fumarolic and diffuse soil degassing west of Mount Epomeo, Ischia, Italy. J. Volcan.Geophys. Res. 133: 291-309.
CHIODINI, G., BALDINI, A., BARBERI, F., CARAPEZZA, M.L., CARDELLINI, C., FRONDINI, F., GRANIERI, D. \& RANALDI, M., 2007: Carbon dioxide degassing at Latera caldera (Italy): Evidence of geothermal reservoir and evaluation of its potential energy.- J. Geophys. Res. 112: B12204. DOI: 10.1029/2006JB004896.

DAVID, M., 1977: Geoestatistical Ore Reserve Estimation, Development in Geomathematics 2.- 364 págs, Elsevier Sci., New York.

DEUTSCH, C. \& JOURNEL, A., 1998: GSLIB: Geostatistical Software Library and Users Guide, [2 ${ }^{\text {nd }}$ ed.].- 369 págs, Oxford University Press, New York.

FARRAR, C., SOREY, M., EVANS, W., HOWLE, J., KERR, B., KENNEDY, B., KING, C. \& SOUTHON, J., 1995: Forest-killing diffuse $\mathrm{CO}_{2}$ emission at Mammoth Mountain as a sign of magmatic unrest.- Nature, 376: 675-678.

FERNÁNDEZ, M., 1990: La actividad del Volcán Poás (Costa Rica): Análisis sísmico durante el periodo 1980-1989.- 185 págs. Univ. de Costa Rica, San José [Tesis Lic.]

FOURNIER, N., RYMER, H., WILLIAMSJONES, G. \& BRENES, J., 2004: High-resolution gravity survey: Investigation of subsurface structures at Poás volcano, Costa Rica.Geophys. Res. Lett., 31: L15602. DOI: 10.1029/2004GL020563.

FRONDINI, F., CHIODINI, G., CALIRO, S., CARDELLINI, C., GRANIERI, D. \& VENTURA, G., 2004: Diffuse $\mathrm{CO}_{2}$ degassing at Vesuvio, Italy.- Bull. Volcanol. 66: 642-651. DOI : $10.1007 / \mathrm{s} 00445-004-$ 0346-x.

GERLACH, T. \& GRAEBER, E., 1985: Volatile budged of Kilawea volcano.- Nature, 313: 273-277. 
GIAMMANCO, S., GURRIERI, S. \& VALENZA, M., 1995: Soil $\mathrm{CO}_{2}$ degassing on Mt Etna (Sicily) during the period 1989-1993: discrimination between climatic and volcanic influences.- Bull. Volcanol., 57: 52-60.

GIGGENBACH, W., 1996: Chemical composition of volcanic gases,- En: SCARPA Y R. TILLING (Eds.): Monitoring and mitigation of volcanic hazards. Springer-Verlag, Berlin-Heidelberg: 221-256.

GRANIERI, D., CARAPEZZA, M., CHIODINI, G., AVINO, R., CALIRO, S., RANALDI, M., RICCI, T. \& TARCHINI, L., 2006: Correlated increase in $\mathrm{CO}_{2}$ fumarolic content and diffuse emission from La Fossa crater (Vulcano, Italy): Evidence of volcanic unrest or increasing gas release from a stationary deep magma body?.- Geophys. Res. Lett. 33: L13316.

GURRIERI, S., LIUZZO, M. \& GIUDICE, G., 2008: Continuous monitoring of soil $\mathrm{CO} 2$ flux on Mt. Etna: The 2004-2005 eruption and the role of regional tectonics and volcano tectonics.- J. Geophys. Res. 113: B09206. DOI: 10.1029/2007JB005003.

HERNÁNDEZ, P., PÉREZ, N., SALAZAR, J., NAKAI, S., NOTSU, K. \& WAKITA, H., 1998: Diffuse emission of carbon dioxide, methane, and helium-3 from Teide volcano, Tenerife, Canary Islands.- Geophs. Res. Lett. 25: 3311-3314.

HERNÁNDEZ, P., NOTSU, K., SALAZAR, J., MORI, T., NATALE, G., OKADA, H., VIRGILI, G., SHIMOIKE, Y., SATO, M. \& PÉREZ, N., 2001a: Carbon dioxide degassing by advective flow from Usu Volcano, Japan.- Science, 292: 83-86.

HERNÁNDEZ, P., SALAZAR, J., SHIMOIKE, Y., MORI, T., NOTSU, K. \& PÉREZ, N., 2001b: Diffuse emission of $\mathrm{CO} 2$ from Miyakejima volcano, Japan.- Chemical Geol. 177: 175-185.
HERNÁNDEZ, P., NOTSU, K., TSURUMI, M., MORI, T., OHNO, M., SHIMOIKE, Y., SALAZAR, J. \& PÉREZ, N., 2003: Carbon dioxide emissions from soils at Hakkoda, north Japan.- J. Geoph. Res. 108(B4): 2210. DOI: 10.1029/2002JB001847.

HERNÁNDEZ, P., NOTSU, K., OKADA, K., MORI, T., SATO, M., BARAHONA, F. \& PEREZ, N., 2006: Diffuse emission of $\mathrm{CO}_{2}$ from Showa-Shinzan, Hollaido, Japan: a sign of volcanic domedegassing.- Pure and Applied Geophysics, 163: 869-881.

HINKLE, M. \& KILBURN, J., 1979: The use of vacutainer tube for collection of soil sample for helium analysis.- USGS Open File Rep. 79: 1441.

HIRABAYASHI, J., OSSAKA, J. \& OZAWA, T., 1986: Geochemical study on volcanic gases at Sakurajima Volcano, Japan.- J. Geophys. Res. 91: 12167-12176.

HOEFS, J., 1980: Stable isotope geochemistry. [2nd ed.].- 1280 págs. Springer-Verlag Berlin.

ITALIANO, F., NUCCIO, M. \& PECORAINO, G., 1998: Steam output from fumaroles of an active volcano: tectonic and magmatic hydrothermal controls on the degassing system at Vulcano (Aeolian arc).- J. Geophys. Res. 103(12): 29829-29841.

LECINSKY, J., HILLEY, G., TOSHA, T., AOYAGI, R., YAMAMOTO, K.\& BENSON, S., 2007: Dynamic coupling of volcanic $\mathrm{CO}_{2}$ flow and wind at the Horseshoe Lake tree kill, Mammoth Mountain, California.- Geophys. Res. Lett. 34: L03401. DOI: 10.1029/2006g1028848.

LOCKE, C., RYMER, H. \& CASSIDY, J., 2003: Magma transfer processes at persistently active volcanoes: insights from gravity observations.- J. Volcanol. Geotherm. Res. 127: 73-86. 
MARTÍNEZ, M., FERNÁNDEZ, E., VALDÉS, J., BARBOZA, V., VAN DER LAAT, R., DUARTE, E., MALAVASSI, E., SANDOVAL, L., BARQUERO, J. \& MARINO, T., 2000: Chemical evolution and volcanic activity of the active crater lake of Poás volcano, Costa Rica, 1993-1997.- J. Volcanol. Geoth. Res. 97: 127-141.

MATSUO, S., 1960: On the origin of volcanic gases.- J. Earth Sci. 8: 222-245.

MATSUO, S., SUZUKI, T., KUSAKABE, M., WADA, H. \& SUZUKI, M., 1975: Isotopic and chemical composition of volcanic gases from Satsuma-Iwojima, Japan.- Geochem. J., 8: 165-173.

McGEE, K. \& GERLACH, T., 1998: Airborne volcanic plume measurements using a FTIR spectrometer, Kilauea volcano, Hawaii.Geophys. Res. Lett. 25(5): 615-618.

MELIÁN, G., GALINDO, I., SALAZAR, J., HERNÁNDEZ, P., PÉREZ, N., RAMÍREZ, C., FERNÁNDEZ, M. \& NOTSU, K., 2001: Spatial and secular variations of diffuse $\mathrm{CO}_{2}$ degassing from Poás Volcano, Costa Rica, Central America.- [Abstract] EOS Trans. Amer. Geophy. Union, 82: F1332.

MELIÁN, G., GALINDO, I., SALAZAR, J., PÉREZ, N., HERNÁNDEZ, P., FERNÁNDEZ, M., RAMÍREZ, C., MORA, R. \& ALVARADO, G., 2002: Hydrogen emissión from Poás volcano, Costa Rica, Central America: a premonitory geochemical signature of volcanic unrest?" - [Resumen] EOS Trans. Amer. Geophys. Union 83: F1489.

MELIÁN, G., MARRERO, R., GALINDO, I., SALAZAR, J., HERNÁNDEZ, P., PÉREZ, N. \& STRAUCH, W., 2003: Monitoring Diffuse carbon dioxide degassing and surface pressure gradient at Cerro Negro vol- cano, Nicaragua. - [Resumen]. EOS, Trans., Am. Geophys. Union 84(46): F1514.

MELIÁN, G., PÉREZ, N., HERNÁNDEZ, P., SALAZAR, J., YOCK, A., SÁNCHEZ, E., ALVARADO, G., SUMINO, H. \& NOTSU, K., 2004: Emisión Difusa de Dióxido de Carbono y Vapor de Mercurio en el volcán Miravalles, Costa Rica.- En: SOTO, G. \& ALVARADO, G. (eds): La Vulcanología y su entorno Geoambiental.Rev. Geol. Amér. Central, 30: 179-188.

MELIÁN, G., GALINDO, I., NOLASCO, D., MARRERO, R., PÉREZ, N., HERNÁNDEZ, P., RAMÍREZ, C., MORA, R., FERNÁNDEZ, M., ALVARADO, G. \& NOTSU, K., 2006: Temporal variations of diffuse $\mathrm{CO}_{2}$ emission from Poás volcano, Costa Rica.- [Abstract] $300^{\text {th }}$ Anniversary Volcano Internacional, GARAVOLCAN. Garachico, Tenerife, 22-27 Mayo de 2006: 25.

MELIÁN, G., GALINDO, I., PÉREZ, N., HERNÁNDEZ, P., FERNÁNDEZ, M., RAMÍREZ, C., MORA R., \& ALVARADO G., 2007: Diffuse emission of hydrogen from Poás volcano, Costa Rica, America Central.- PAGEOPH Special Issue "Terrestrial Fluids, Earthquakes and Volcanoes: The Hiroshi Wakita vol. II", 164(12): 2465-2487. DOI: 10.1007/ s00024-007-0282-8.

MELIÁN, G., 2008: Emisión difusa de dióxido de carbono y otros volátiles en el volcán Poás, Costa Rica, América Central.- 401 págs. Univ. La Laguna (ULL), España [Tesis Ph.D.].

MINAKAMI, T., 1969: Earthquake originating from volcanoes.- [Abstract] XVIII Covn. ASS. Geof. Ital., Napoli 1-4 October: 158-169.

MORA, R., RAMÍREZ, C. \& FERNÁNDEZ, M., 2004: La actividad de los volcanes de la Cordillera Central, 1998-2002, Costa Rica. - 
En: SOTO, G.J. \& ALVARADO, G.E. (eds): La Vulconología y su entorno Geoambiental.Rev. Geol. Amér. Central, 30: 189-197.

NOLASCO D., MELIÁN, G., GALINDO, I., SALAZAR, J., PÉREZ, N., HERNÁNDEZ, P., FERNÁNDEZ, M., RAMÍREZ, C., MORA, R. \& ALVARADO, G., 2003: Diffuse mercury degassing at Poás Volcano, Costa Rica, Central America.- [Abstrct] EOS Trans. Amer. Geophys. Union 84 (46): F11513.

NOTSU K., SUGIYAMA K., HOSOE M., UEMURA A., SHIMOIKE Y., TSUNOMORI F., SUMINO H., YAMAMOTO J., MORI T. \& HERNANDEZ P., 2005: Diffuse $\mathrm{CO}_{2}$ efflux from Iwojima volcano, Izu-Ogasawara arc, Japan.- J. Volcanol. Geother. Res., 139: 147-161.

NOTSU, K., MORI, T., CHANCHAH DO VALE, S., KAGI, H.ANDITO, T., 2006: Monitoring quiescent volcanoes by diffuse $\mathrm{CO}_{2}$ degassing: case study of Mt. Fuji, Japan.- Pure Appl. Geophys., 163: 825-835.

PADRÓN, E., HERNÁNDEZ, P., TOULKERIDIS, T., PÉREZ, N., MARRERO, R., MELIÁN, G., VIRGILI, G.\& NOTSU, K., 2008: Diffuse $\mathrm{CO}_{2}$ emission rate from Pululahua and the lake-filled Cuicocha calderas, Ecuador.- J. Volcanol. Geother., 176: 163-169. DOI: 10.1016/j. jvolgeores.2007.11.023.

PARKINSON, K., 1981: An improved method for measuring soil respiration in the field.- J. Applied Ecol. 18: 221-228.

PASTERNACK, G. \& VAREKAMP, J., 1997: Volcanic lake systematics.- Bull. Volcanol. 58: 528-538.

PÉREZ, N., 1992: Estudio Geoquímico de las Descargas Volcánoco-MagmáticoHidrotermales de la Caldera de Rabaul,
Papua Nueva Guinea.- 471 págs .Univ. de La Laguna, España [Tesis Ph.D.].

PÉREZ, N., WAKITA, H., LOLOK, D., PATIA, H., TALAI, B. \& McKEE, C., 1996: Anomalous soil gas $\mathrm{CO}_{2}$ concentrations and relation to seismic activity at Rabaul Caldera, Papua New Guinea.- Geogaceta 20: 1.000-1.003.

PÉREZ, N., SALAZAR, J., SABALLOS, A., ÁlVAREZ, J., SEGURA, F., HERNÁNDEZ, P. \& NOTSU, K., 2000: Diffuse degassing of $\mathrm{CO} 2$ from Masaya caldera, Central America.- [Abstract] EOS Trans. AGU, 81: F1345.

PÉREZ, N., SALAZAR, J., HERNÁNDEZ, P., SORIANO, T., LOPEZ, K. \& NOTSU, K., 2004: Diffuse $\mathrm{CO}_{2}$ and ${ }^{222} \mathrm{Rn}$ degassing from San Salvador volcano, El Salvador, Central America.- Bull. Geolog. Soc. Amer., 375: 227-236.

PÉREZ N., HERNÁNDEZ P., PADRÓN, E., CARTAGENA, R., OLMOS, R., BARAHONA, F., MELIÁN, G., SALAZAR, P. \& LÓPEZ, D., 2006: Anomalous diffuse $\mathrm{CO}_{2}$ emission prior to the January 2002 short-term unrest at San Miguel volcano, El Salvador, Central America.- Pure Appl. Geophys., 163(4): 883-896.

PÉREZ, N. \& HERNÁNDEZ, P., 2007: Earthquake forecasting research in active volcanic areas by means of diffuse $\mathrm{CO}_{2}$ emission studies.- En: SEN, P. \& DAS, K (eds): Geochemical Precursors for Earthquakes.- MacMillan India Ltd.: 94-103.

RIZZO A., GRASSA, F., INGUAGGIATO, S., LIOTTA, M., LONGO, M., MADONIA, P., BRUSCA, L., CAPASSO, G., MORICI, S., ROUWET, D. \& VITA F., 2009: Geochemical evaluation of observed changes in volcanic activity during 
the 2007eruption at Stromboli (Italy).- J. Volcanol. Geotherm. Res. 182: 246-254.

ROWE, G., BRANTLEY, S., FERNÁNDEZ, M., FERNÁNDEZ, J., BARQUERO, J. \& BORGIA, A., 1992a: Fluid-volcano interacting in an active stratovolcano: the Crater Lake system of Poás volcano, Costa Rica.J. Volcanol. Geotherm. Res. 49: 23-51.

ROWE, G., OHSAWA, S., TAKANO, B., BRANTLEY, S., FERNÁNDEZ, J. \& BARQUERO, J., 1992b: Using Crater Lake chemistry to predict volcanic activity at Poás volcano, Costa Rica.- Bull. Volcanol. 54: 494-503.

RYMER, H. \& BROWN, G., 1989: Gravity changes as a precursor to volcanic eruption at Poás volcano, Costa Rica.- Nature, 342: 902-905.

RYMER, H., CASSIDY, J., LOCKE, C., BARBOZA, M., BARQUERO, J., BRENES, J. \& VAN DER LAAT, R., 2000: Geophysical studies of the recent 15-year eruptive cycle at Poás volcano, Costa Rica.- J. Volcanol. Geotherm. Res. 97: 425-442.

RYMER, H., LOCKE, C., BRENES, J. \& WILLIAMS-JONES, G., 2005: Magma plumbing processes for persistent activity at Poás volcano.- Geophys. Res. Lett. 32: L08307. DOI: 10.1029/2004GL22284.

SALAZAR, J., HERNÁNDEZ, P., PÉREZ, N., MELIÁN, G., ÁlVAREZ, J., SEGURA, F. \& NOTSU, K., 2001: Diffuse emission of carbon dioxide from Cerro Negro Volcano, Nicaragua.- Geoph. Res. Lett. 28: 4275-4278.

SALAZAR, J., HERNÁNDEZ, P., PÉREZ, N., OLMOS, R., BARAHONA, F., CARTAGENA, R., SORIANO, T., LOPEZ, K. \& NOTSU, K., 2003: Spatial and temporal variations of diffuse $\mathrm{CO}_{2}$ de- gassing at Santa Ana-Izalco-Coatepeque volcanic complex, El Salvador, Central America.- Bull. Geolog. Soc. Amer. Special, 375: 135-146.

SANO, Y., NOTSU, K., ISHIBASHI, J., IGARASHI, G. \& WAKITA, H., 1991: Secular variations in helium isotope ratios in an active volcano: eruption and plug hypothesis.- Earth Planet. Sci. Lett. 107: 95-100.

SATO, M., 1988: Continuous monitoring of hydrogen in volcanic areas: petrological rationale and early experiments.- Rendiconti Della Società Italiana Di Mineralogia Petrologia, 43: 1.265-1.281.

SCHWARCZ, H. 1969: Carbon isotopes in nature.In: WEDEPOHL, K.M. (ed.): Handbook of Geochemistry, II/I, 6-B-1 - 6-B-15.

SINCLAIR, A., 1974: Selection of thresholds in geochemical data using probability graphs.- J. Geochem. Explor., 3: 129-149.

SYMONDS, R., ROSE, W., GERLACH, T., BRIGGS, P. \& HARMON, R., 1990: Evaluation of gases, condensates, and $\mathrm{SO} 2$ emissions from Augustine volcano, Alaska: the degassing of a Cl-rich volcanic system.- Bull. Volcanol. 52: 355-374.

TENNANT, C. \& WHITE, M., 1959: Study of the distribution of some geochemical data.Econ. Geol. 54: 1281-1290.

VASELLI, O., TASSI, F., MINISSALE, G., MONTEGROSSI, G., DUARTE E., FERNANDEZ E. \& BERGAMASCHI, F., 2003: Fumarole migration and fluid geochemistry at Poás Volcano (Costa Rica) from 1998 and 2001.- En: OPPENHEIMER, C., PYLE D., \& BARCLAY. J. (Eds).- Volcanic Degassing. Geological Society, London, Special Publication, 213: 247-262, DOI: 10.1144/ GSL.SP.2003.213.01.15. 
ZIMMER, M., FISCHER, D., ALVARADO, G., SHARP, Z. \& WALKER, J., 2004: Nitrogen systematic and gas fluxes of sub- duction zones: Insights from Costa Rica arc volatiles.- Geochem. Geoph. Geosyst. 5: $1.525-2.027$. 